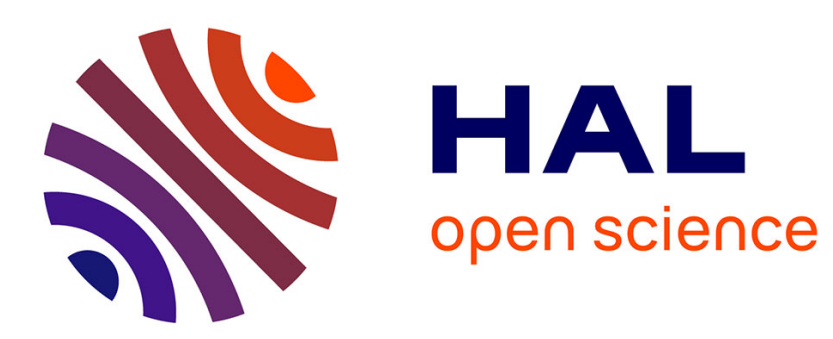

\title{
Évaluation de l'activité des dermatologues 5 à 7 ans après la fin de leur internat
}

Nesrine Bouyahiaoui Zitouni

\section{To cite this version:}

Nesrine Bouyahiaoui Zitouni. Évaluation de l'activité des dermatologues 5 à 7 ans après la fin de leur internat. Dermatologie. 2019. dumas-02459106

\section{HAL Id: dumas-02459106 https://dumas.ccsd.cnrs.fr/dumas-02459106}

Submitted on 29 Jan 2020

HAL is a multi-disciplinary open access archive for the deposit and dissemination of scientific research documents, whether they are published or not. The documents may come from teaching and research institutions in France or abroad, or from public or private research centers.
L'archive ouverte pluridisciplinaire HAL, est destinée au dépôt et à la diffusion de documents scientifiques de niveau recherche, publiés ou non, émanant des établissements d'enseignement et de recherche français ou étrangers, des laboratoires publics ou privés. 


\title{
UNIVERSITÉ DE PICARDIE JULES VERNE
}

ANNÉE 2019

$\mathbf{N}^{\circ} 2019$ - 134

\section{Évaluation de l'activité des dermatologues 5 à 7 ans après la fin de leur internat.}

\author{
THÈSE POUR LE DOCTORAT EN MÉDECINE \\ (DIPLOME D’ÉTAT)
}

\author{
Présentée et soutenue \\ Le 4 octobre 2019 \\ Par Nesrine BOUYAHIAOUI épouse ZITOUNI
}

PRÉSIDENT DU JURY : Madame le Professeur LOK

MEMBRES DU JURY : Monsieur le Professeur DEVAUCHELLE Monsieur le Professeur GANRY Monsieur le Professeur GIGNON

DIRECTEUR DE THÈSE : Monsieur le Docteur ARNAULT 
À mon président du jury,

\section{Madame le Professeur Catherine LOK}

Professeur des Universités - Praticien Hospitalier

Dermatologie - Vénéréologie

Assesseur du $3^{\text {ème }}$ cycle

Chef de Service de Dermatologie

Chef du Pôle des 5 Sens

Vous me faites l'honneur de présider mon jury de thèse. Je vous remercie de votre confiance, de votre soutien, de votre disponibilité et des précieux conseils pour la réalisation de ce travail. Je vous prie d'accepter l'expression de mon plus profond respect et le témoignage de ma sincère reconnaissance. 
Aux membres du jury,

\section{Monsieur le Professeur Bernard DEVAUCHELLE}

Professeur des Universités - Praticien Hospitalier

Chirurgie maxillo-faciale

Pôle des 5 Sens

Docteur Honoris Causa de l'Université de Louvain Belgique

Chevalier dans l'Ordre de la Légion d'Honneur

Commandeur dans l'Ordre des Palmes Académiques

Officier dans l'Ordre National de la Légion d'Honneur

Vous êtes pour moi une référence et c'est un immense honneur que vous me faites de juger ma thèse. Je vous en remercie sincèrement et vous prie de croire en mon plus profond respect.

\section{Monsieur le Professeur Olivier GANRY}

Professeur des Universités - Praticien Hospitalier

Épidémiologie, économie de la santé et Prévention

Responsable du service d'Épidémiologie, hygiène hospitalière et santé publique

Pôle «Biologie, pharmacie et santé des population »

Vous me faites l'honneur de juger mon travail. Honorée de votre présence, je vous remercie de l'intérêt que vous y portez et vous prie d'accepter l'expression de ma vive reconnaissance. 


\section{Monsieur le Professeur Maxime GIGNON}

Professeur des Universités - Praticien Hospitalier

Épidémiologie, hygiène hospitalière et santé publique

Vous avez tout de suite accepté de juger ce travail qui vous a intéressé. Je vous remercie sincèrement de l'enthousiasme dont vous avez fait preuve et vous adresse ici l'expression de ma plus grande considération.

\section{A mon directeur de thèse,}

\section{Monsieur le Docteur Jean Philippe ARNAULT}

Praticien Hospitalier

Dermatologie - Vénéréologie

Il n'y a pas de mots pour exprimer toute la gratitude et la reconnaissance que j'éprouve à ton égard. Merci de m'avoir autant appris, tu as été comme un mentor pour moi durant tout cet internat. Merci de tout le temps que tu as passé avec moi pour la correction des cas clinique de DES, de mon article et finalement de ma thèse. 


\section{Remerciements personnels :}

\section{À Selim, mon mari, mon meilleur ami, mon tout,}

Tu es ce qui m'est arrivé de mieux dans la vie. Merci mille fois de ton soutien durant tout mon internat. Tu m'as toujours encouragée et poussée à donner le meilleur de moi-même pour réussir. Merci de ta patience pour m'avoir supportée depuis le début et bon courage pour me

supporter pendant encore longtemps hahaha. Je sais que tu es fier de moi et c'est mon plus beau cadeau dans la vie. Ils disent que tous les chemins mènent à Rome... En tout cas, tous les miens mènent à toi. Quel honneur de marcher à ton bras ! Et quel bonheur aussi... La vie est si belle assise à tes côtés, je te serai fidèle même de l'autre côté.

\section{À ma mère, la meilleure femme sur terre,}

Que dire ? Les larmes me montent rien qu'à l'idée de tout ce que tu as fait pour moi. Tu m'as tout donné, je ne pourrai jamais te remercier assez. J'espère juste que tu es fière d'avoir une fille comme moi et d'assister aujourd'hui à ma thèse, l'accomplissement de tout mon parcours scolaire. Merci infiniment. Je t'aime démesurément.

\section{À mon père, ce grand homme digne,}

Tu m'as toujours poussée à travailler, à étudier, à me dépasser pour être parmi les meilleurs. Je suis fière aujourd'hui de me tenir devant toi en robe de thèse et de te montrer que tu as réussi, que tous tes efforts investis en moi n'ont pas été vains.

\section{À mes frère et sœur, ces grosses têtes salées,}

On a vraiment été comme les 3 mousquetaires : souvent à se disputer mais au final toujours là les uns pour les autres dans les moments importants. Je vous aimerai toute ma vie et serai toujours là pour vous. Je ne suis certainement pas la meilleure grande sœur du monde mais je donnerai ma vie pour vous.

\section{À toutes mes amies/sœurs, les perles de ma vie,} Merci pour tous ces merveilleux moments de rigolades, de déconnages, de pétages de plomb intensifs. Impossible d'oublier. Vous avez marqué ma vie à jamais. J'ai des goûts de luxe donc des amies en or. Vous puez toutes le chat, même après toutes ces années... 


\title{
À So3ad, cette vieille meuf que personne ne peut remplacer,
}

Oui même pour ma thèse, j'écris ton prénom comme ça ! Ce sont mes remerciements je fais un peu ce que je veux ! Merci pour ces milliers d'excellents moments pendant nos études, merci pour ces fous-rire incessants, pour ces années de pur bonheur et de joie à tes côtés. Les inséparables, disaient-ils de nous.

\begin{abstract}
À Ludivine, Coup de foudre instantané, tu es rapidement devenue comme un membre de ma famille. Je n'oublierai jamais tout ce que tu as fait pour moi ni nos centaines de bons souvenirs ensemble. Je te souhaite le meilleur et tout le bonheur du monde.
\end{abstract}

\section{À toute l'équipe de dermatologie,}

Mes co-internes de dermatologie et de rhumatologie (et ma Julia Sanchez), les infirmières, les secrétaires, Patricia et les chefs, je me suis régalée auprès de vous pendant ces années. Je n'en garderai que le meilleur. J'ai hâte de revenir parmi vous pour la suite des aventures.

\section{Mention spéciale pour Thomas,}

Mon maître Yoda, je me suis accrochée à toi tel à un koala à son arbre pendant tout l'internat. Tu m'as tellement apporté, tellement appris. Merci infiniment, c'était un réel plaisir d'apprendre de toi et de faire mon mémoire avec tes précieux conseils.

\section{Au reste de ma famille,} À tous les BOUYAHIAOUI d'Algérie et d'ailleurs, les FELLAH et même les ZITOUNI, sachez tous que c'est un honneur de faire partie de votre famille et que j'ai toujours été au bout de moi-même dans la vie pour être digne de vous.

\section{À papi Hmida, Mani, Baba Moh et tonton Nacer,}

De là où vous êtes, je pense à vous. Vous auriez sûrement été tous très fiers de me voir aujourd'hui. J'ai bien grandi, rassurez-vous.

À tous, je vous dédie cette thèse, j'espère qu'elle va vous plaire

J'y ai mis tout mon cœur, mon envie, mon savoir-faire. 


\section{RÉSUMÉ}

Introduction : D'après les rapports du CNOM (Conseil National de 1'Ordre des Médecins), le nombre de dermatologues est en diminution (3821 en janvier 2007, 3485 en 2017 et 3328 en janvier 2018). Le mode d'exercice des dermatologues évolue avec une baisse de 7,3\% de l'exercice libéral de 2009 à 2015. Nous avons cherché à évaluer l'activité des jeunes dermatologues en France, aucune donnée n'étant disponible à l'heure actuelle.

Matériel et Méthodes : Il s'agit d'une enquête observationnelle transversale nationale interrogeant les dermatologues ayant fini leur internat depuis 5 à 7 ans avec l'aide du CEDEF (Collège des Enseignants de DEermatologie de France) qui a fourni les listes de noms et les adresses mail. Un questionnaire anonyme de 30 questions leur a été envoyé par mail sur les caractéristiques de la population, leur statut et mode d'exercice et leur pratique quotidienne.

Résultats : Sur un total de 188 dermatologues, 172 ont pu être joints et 83 ont répondu. 93\% étaient des femmes avec un âge moyen de 33,4 ans. $78 \%$ ont fait un post-internat. 54\% exerçaient dans une ville de CHU (Centre Hospitalier Universitaire) et seulement $17 \%$ en « désert médical ». La durée moyenne de remplacements était de 2,8 ans avant installation. 50\% faisaient déjà ou prévoyaient prochainement de faire de la télémédecine. $42 \%$ étaient libéraux dont $15 \%$ de remplaçants, $29 \%$ salariés et $29 \%$ en activité mixte. L'activité s'étalait sur 8 demi-journées/semaine en moyenne dont une de chirurgie pour $94 \%$ et une de laser pour $40 \%$. $47 \%$ pratiquaient de la photothérapie et $43 \%$ de la PDT (PhotoThérapie Dynamique). 18\% faisaient de 1'esthétique et pour 7\% elle représentait plus de $50 \%$ de leur activité. Parmi les libéraux, 57\% étaient en secteur 2, 37\% en secteur 1 et $6 \%$ non conventionnés et seulement $28 \%$ s'installaient seuls. Les freins à l'exercice libéral étaient : difficultés de gestion d'une entreprise $(60 \%)$, craintes des charges $(54 \%)$, manque d'information $(46 \%)$ et de formation à la pratique libérale $(28 \%)$, manque financier $(13 \%)$.

Discussion : Le nombre de dermatologues diminue alors que le nombre de médecins augmente. Les jeunes dermatologues ne compensent pas les départs en retraite. La dermatologie est une spécialité qui se féminise et encore plus rapidement que la médecine en général. L'évolution du mode d'exercice se fait vers le développement du secteur 2, la diversification d'une activité de laser et d'esthétique, ainsi que vers l'exercice salarié ou mixte au détriment de l'activité libérale exclusive. L'installation se fait en association plutôt que seul et les inégalités territoriales de densités se creusent.

Conclusion : L'étude de l'activité des dermatologues de notre population confirme une évolution du mode d'exercice par rapport aux générations antérieures. 


\section{ABBRÉVIATIONS}

AAD : American Academy of Dermatology

ADEC : Académie de Dermatologie Esthétique et Correctrice

CADEC : Cours d'Anatomie appliqué à la Dermatologie Esthétique et Correctrice

CARD : Congrès Annuel de Recherche en Dermatologie

CCAM : Classification Commune des Actes Médicaux

CEDEF : Collège des Enseignants de DEermatologie de France

CHU : Centre Hospitalier Universitaire

CNAM : Caisse Nationale d'Assurance Maladie

CNOM : Conseil National de l'Ordre des Médecins

DES : Diplôme d'Études Spécialisées

DPC : Développement Professionnel Continu

DU/DIU : Diplôme Universitaires/Inter-Universitaires

EADV : European Academy of Dermatology and Venereology

ECN : Épreuves Classantes Nationales

ESDR : European Society of Dermatological Research

FFFCEDV : Fédération Française de Formation Continue et d'Évaluation en

Dermatologie Vénéréologie

gDEC : groupe de Dermatologie Esthétique et Correctrice

GERDA : Groupe d'Études et de Recherche en Dermato-Allergologie

INSERM : Institut National de la Santé et de la Recherche Médicale

IPL : Lumière intense pulsée

JDP : Journées de Dermatologie Parisiennes

JIRD : Journées Interactives de Réalités thérapeutiques en Dermatologie

JNPD : Journées Nationales Provinciales de Dermatologie

LED : Light Emitting Diode

PDT : PhotoThérapie Dynamique

SFD : Société Française de Dermatologie

SNDV : Syndicat National des Dermatologues Vénéréologues

UV : Ultra-Violet 


\section{Table des matières}

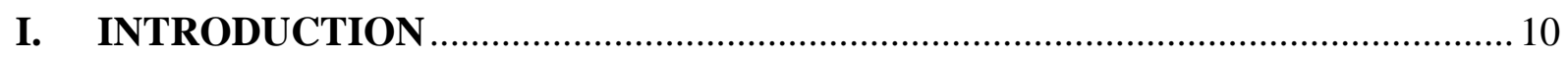

1. La démographie dermatologique en France ……………………............................. 10

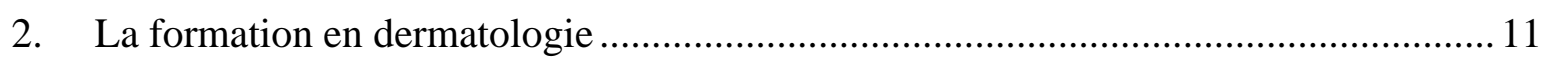

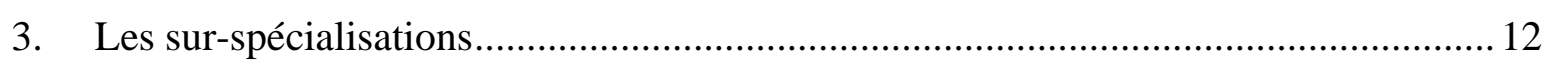

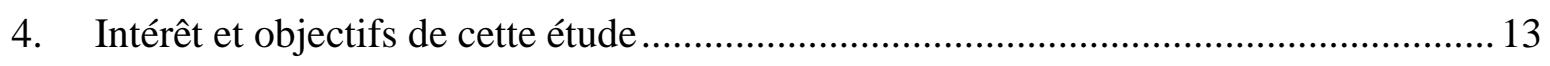

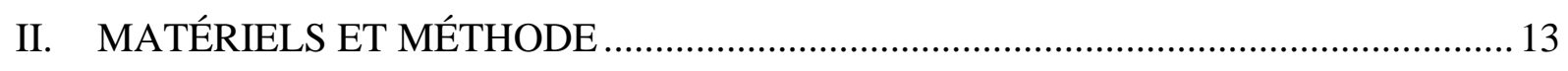

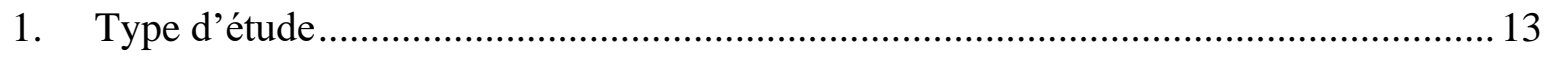

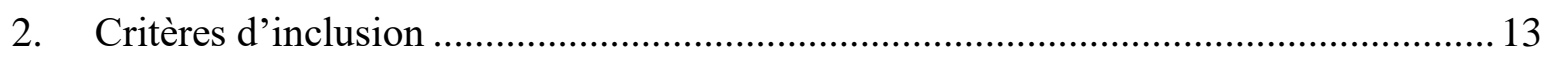

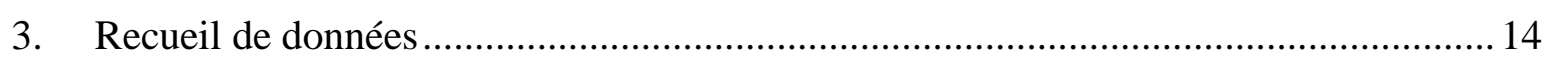

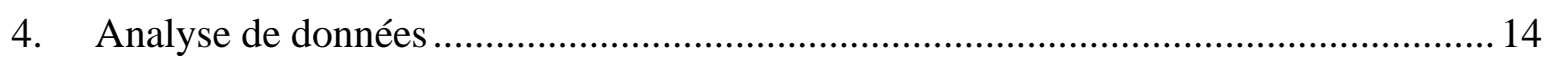

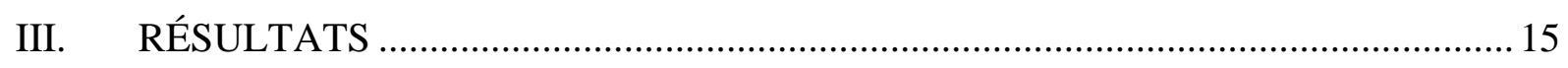

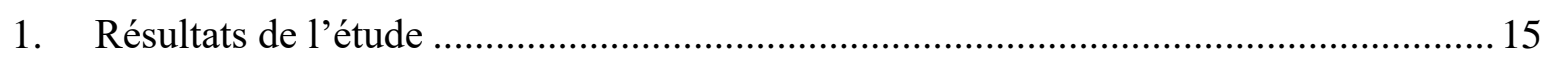

2. Comparaison de l'activité actuelle avec la formation reçue pendant l'internat ...........24

3. Comparaison avec la population de tous les dermatologues français ............................27

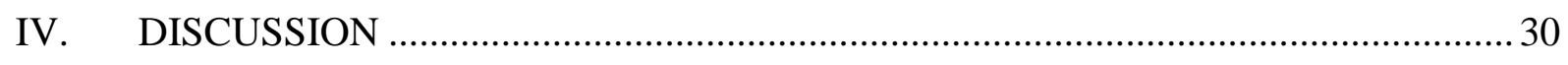

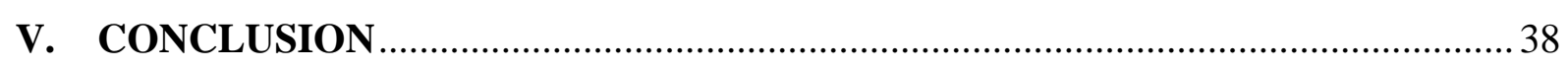

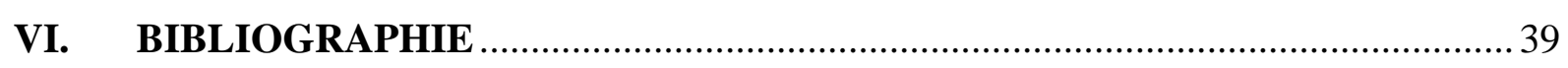

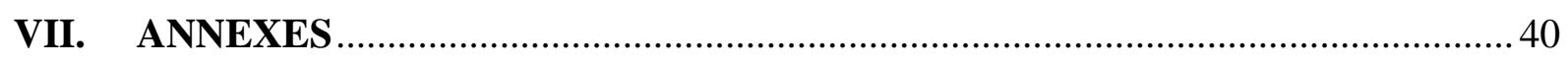

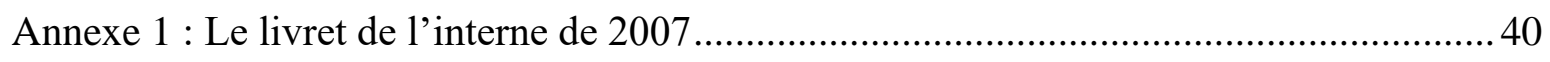

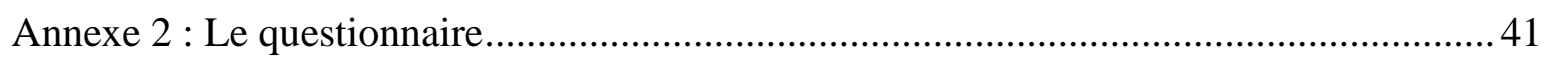




\section{INTRODUCTION}

\section{La démographie dermatologique en France}

D'après les rapports du CNOM (Conseil National de l'Ordre des Médecins), l'effectif des médecins spécialistes augmente régulièrement mais le nombre de dermatologues est en diminution (3821 en janvier 2007, 3485 en 2017 et 3328 en janvier 2018). Seuls 15\% d'entre eux ont moins de 40 ans et $34 \%$ plus de 60 ans (1).

Les dermatologues ne sont pas répartis de façon régulière sur le territoire français et sont plutôt concentrés dans les régions de CHU (Centre Hospitalier Universitaire) (figure 1).

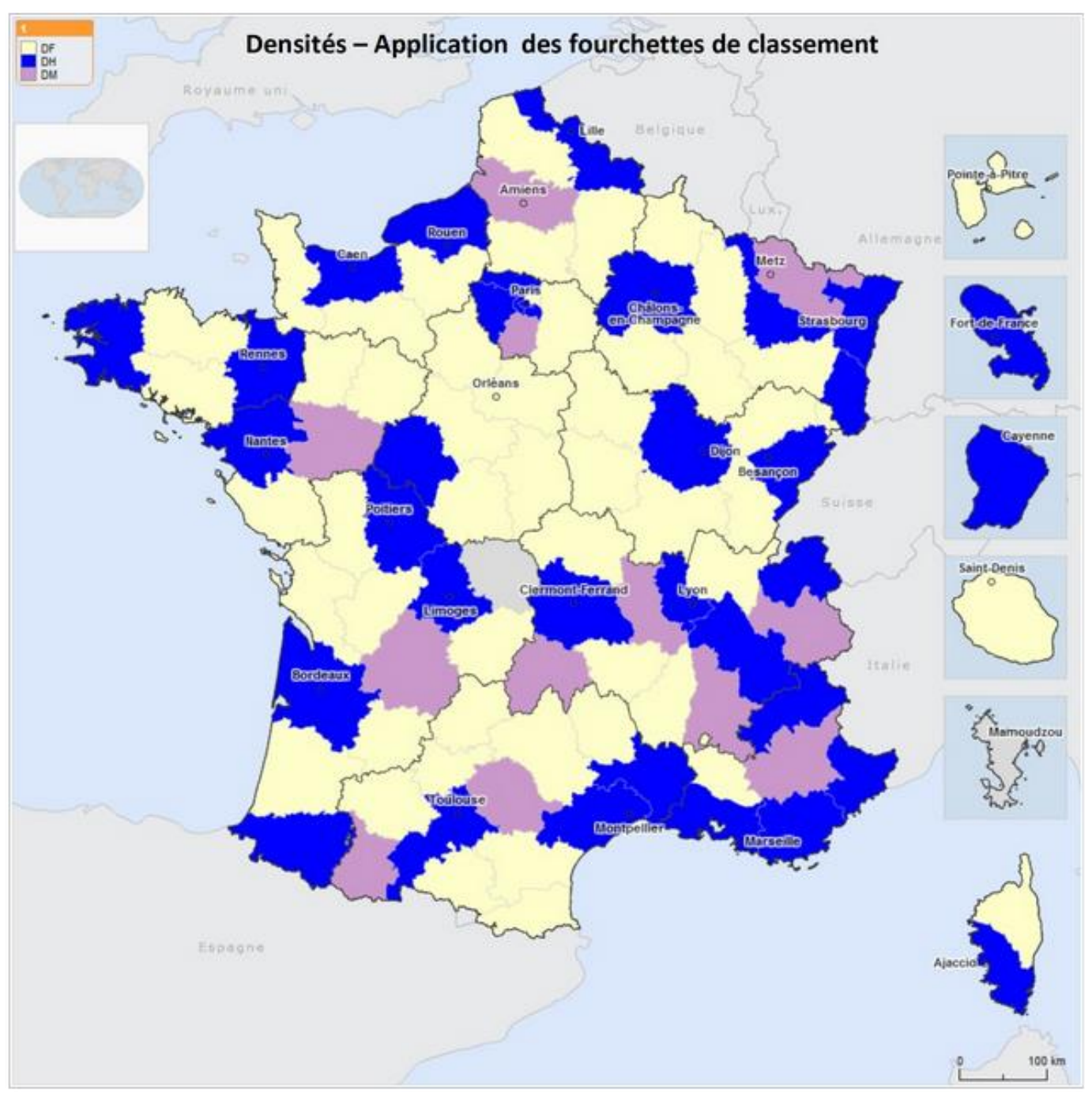

Figure 1. Répartition des densités de dermatologues sur le territoire français (1).

$\mathrm{DF}=$ Densité Faible en beige $; \mathrm{DM}=$ Densité Moyenne en rose $; \mathrm{DH}=$ Densité Haute en bleu. 
D'après l'atlas de la démographie médicale en France de 2015 du CNOM, la dermatologie fait partie des 4 spécialités (avec la rhumatologie, la chirurgie générale et l'ORL) dont le mode d'exercice en libéral diminue le plus avec une baisse de $7.3 \%$ de 2007 à 2015 (2).

\section{La formation en dermatologie}

Le premier cycle en $3^{\text {ème }}$ année de médecine comprend une unité d'enseignement sémiologique sur le revêtement cutané et au $2^{\text {ème }}$ cycle ( $5^{\text {ème }}$ année) des cours de pathologie dermatologique. Le référentiel des cours est édité par le Collège des Enseignants de DErmatologie de France (CEDEF).

L'internat de dermatologie dure 4 ans. Les internes se réfèrent au livret de l'interne. Il est réalisé par le CEDEF et remis à jour régulièrement.

La formation médicale continue (FMC) est variée et résumée dans le tableau 1. 


\begin{tabular}{|c|c|}
\hline Congrès nationaux & $\begin{array}{l}\text { JDP (Journées Dermatologiques Parisiennes), GERDA (Groupe d'Études et } \\
\text { de Recherche en Dermato-Allergologie), photodermatologie, congrès annuel } \\
\text { de recherche dermatologique (CARD), SNDV (Syndicat National des } \\
\text { Dermatologues Vénéréologues) }\end{array}$ \\
\hline $\begin{array}{l}\text { Congrès } \\
\text { internationaux }\end{array}$ & $\begin{array}{l}\text { EADV (European Academy of Dermatology and Venereology), ESDR } \\
\text { (European Society of Dermatological Research) et AAD (American } \\
\text { Academy of Dermatology) }\end{array}$ \\
\hline Livres & $\begin{array}{l}\text { Saurat (J-H SAURAT), Dermatologie : de la clinique à la microscopie (B. } \\
\text { CRIBIER), Dermatologie infectieuse (M. MOKNI), Atlas de poche de } \\
\text { dermatologie (M. RÖCKEN), Dermatologie : l'essentiel (L. JEAN et } \\
\text { BOLOGNIA) }\end{array}$ \\
\hline $\begin{array}{l}\text { DU/DIU : Diplômes } \\
\text { (inter)universitaires }\end{array}$ & $\begin{array}{l}\text { Techniques d'injection et de volumétrie, muqueuse buccale, maladies } \\
\text { systémiques, dermatopédiatrie, esthétique/cosmétologie/lasers, } \\
\text { immunothérapies ciblées, cuir chevelu, dermoscopie, dermatologie } \\
\text { chirurgicale }\end{array}$ \\
\hline $\begin{array}{l}28 \text { sous-groupes de la } \\
\text { SFD (Société } \\
\text { Française de } \\
\text { Dermatologie) dont } \\
\text { voici quelques-uns }\end{array}$ & $\begin{array}{l}\text { Cancérologie cutanée, dermato-allergologie, bulle, éducation thérapeutique } \\
\text { en dermatologie, lymphomes cutanés, dermatopathologie, peau noire, } \\
\text { toxidermie, psychodermatologie, ongle, recherche sur le psoriasis, urticaire, } \\
\text { dermatologie pédiatrique, gDEC (groupe de Dermatologie Esthétique et } \\
\text { Correctrice) }\end{array}$ \\
\hline Séminaires & $\begin{array}{l}\text { Dermatopathologie, dermatologie chirurgicale, dermatologie esthétique et } \\
\text { correctrice de l'ADEC (Académie de Dermatologie Esthétique et } \\
\text { Correctrice), IST (Infections Sexuellement Transmissibles), pathologies des } \\
\text { muqueuses, laser, sémiologie et thérapeutique dermatologique, Cours } \\
\text { d'Anatomie appliqué à la Dermatologie Esthétique et Correctrice (CADEC), } \\
\text { oncodermatologie, angiodermatologie }\end{array}$ \\
\hline $\begin{array}{l}\text { Journées de } \\
\text { formation }\end{array}$ & $\begin{array}{l}\text { JNPD (Journées Nationales Provinciales de Dermatologie), JIRD (Journées } \\
\text { Interactives de Réalités thérapeutiques en Dermatologie), journées } \\
\text { cicatrisations annuelles, colloque annuel rhumatologi-dermatologie, journée } \\
\text { des } 4 \text { saisons de la SFD }\end{array}$ \\
\hline Sites internet & $\begin{array}{l}\text { Banques iconographiques, cas clinique, quizz interactifs, bibliographie, } \\
\text { recommandations }\end{array}$ \\
\hline
\end{tabular}

Tableau 1. Outils de formation médicale continue.

\section{Les sur-spécialisations}

Plusieurs sur-spécialités sont à la portée du dermatologue : l'allergologie, l'angiologie ou médecine vasculaire avec les malformations vasculaires, la chirurgie dermatologique, la médecine esthétique, les manifestations dermatologiques des maladies systémiques, les lasers, les pathologies de la muqueuse buccale, l'oncologie, l'onychologie, la peau noire, la pédiatrie, le psoriasis, la photothérapie dynamique, UVA (Ultra-Violet A) et UVB (Ultra-Violet B), la trichologie, les troubles pigmentaires, les troubles trophiques des membres inférieurs et la vénéréologie et pathologies de la muqueuse génitale. 
4. Intérêt et objectifs de cette étude

Chaque année, environ 90 internes finissent leur internat de dermatologie et vénérologie en France (3). Aucune donnée n'existe sur la démographie des jeunes dermatologues de France et leur activité.

Notre étude a pour objectif d'évaluer la pratique des dermatologues 5 à 7 ans après la fin de leur internat. Les objectifs secondaires sont de comparer leur activité actuelle avec l'enseignement qu'ils ont reçu pendant leur internat et de comparer cette population à l'ensemble des dermatologues français. Pour notre population cible, nous avons choisi d'utiliser le livret de l'interne réalisé en juin 2007 (annexe 1) comme référence de la formation reçue pendant l'internat.

\section{MATÉRIELS ET MÉTHODE}

\section{Type d'étude}

Il s'agit d'une enquête transversale, nationale et observationnelle.

\section{Critères d'inclusion}

Cette étude s'intéresse aux dermatologues ayant fini leur internat depuis 5 à 7 ans. Autrement dit, les dermatologues ayant fait leur internat entre 2008 à 2012 (pour ceux ayant fini depuis 7 ans) et entre 2010 et 2014 (pour ceux ayant fini depuis 5 ans). Ainsi, était incluse toute personne figurant sur la liste ECN (Épreuves Classantes Nationales) des internes en dermatologie en France de 2008, 2009 et 2010. À notre connaissance, il n'existe pas de liste exhaustive antérieure à 2008. Ces listes, comportant les noms, prénoms, faculté de médecin d'origine, année de passage de l'ECN et adresses mail, nous ont été fournies par le CEDEF après requête. 
3. Recueil de données

Un questionnaire de type Google Forms de 30 questions (annexe 2) leur a été envoyé par mail en leur expliquant que les données recueillies l'étaient de façon anonyme.

Les questions portaient sur :

- Les caractéristiques de la population : âge, sexe, post-internat, remplacements

- Leur cursus et formations

- Leurs outils de formations actuels

- Leur activité actuelle : lieu, techniques, sur-spécialisations

- Leur pratique libérale ou non : les difficultés rencontrées, les freins.

La $29^{\text {ème }}$ question leur proposait de recevoir les résultats de cette étude s'ils le souhaitaient et le cas échéant, ils devaient y écrire leur adresse mail et ainsi renoncer à leur anonymat.

La $30^{\text {ème }}$ question était ouverte avec la possibilité de laisser des commentaires libres, de dire ce qu'ils leur avaient manqué pendant leur internat, ce qu'ils pensaient de la démographie des jeunes dermatologues etc.

Les données concernant la démographie dermatologique française ont été récupérées d'une étude réalisée par la FFFCEDV (Fédération Française de Formation Continue et d'Evaluation en Dermatologie Vénéréologie) fin 2018. Cette étude concernait tous les dermatologues français et les données ont été recueillies par un questionnaire. Elle n'a pas encore été publiée mais des résultats préliminaires ont été présentées lors des JNPD (Journées Nationales Provinciales de Dermatologie) mars 2019.

4. Analyse de données

Les données recueillies à partir du questionnaire étaient analysées de façon descriptive grâce à l'utilisation des logiciels Google Forms ${ }^{\circledR}$ et Microsoft Excel®.

L'expression des résultats a été réalisée en pourcentages, en nombres entiers et en moyennes. 


\section{RÉSULTATS}

\section{Résultats de l'étude}

Parmi les 207 internes figurant sur ces listes, 19 ont été exclus :

- 4 ayant fait un droit au remord pour hématologie, médecine interne, gastroentérologie et médecine générale

- 2 étant médecins généralistes s'étant retrouvés sur les listes en ayant fait leur premier semestre d'internat dans un service de dermatologie

- 13 ayant fini leur internat depuis moins de 5 ans (leur internat ayant donc duré plus de 4 ans pour cause d'année de recherche, de Master II, de mise en disponibilité, de grossesse etc.)

Dans la population de 188 dermatologues concernés restants, 16 n'ont pas pu être joints (adresse mail non valide). Nous avons finalement recueilli 83 réponses sur les 172 participants, soit environ $48 \%$ (figure 2). Ce qui correspondait à 44\% des 188 dermatologues initiaux. 


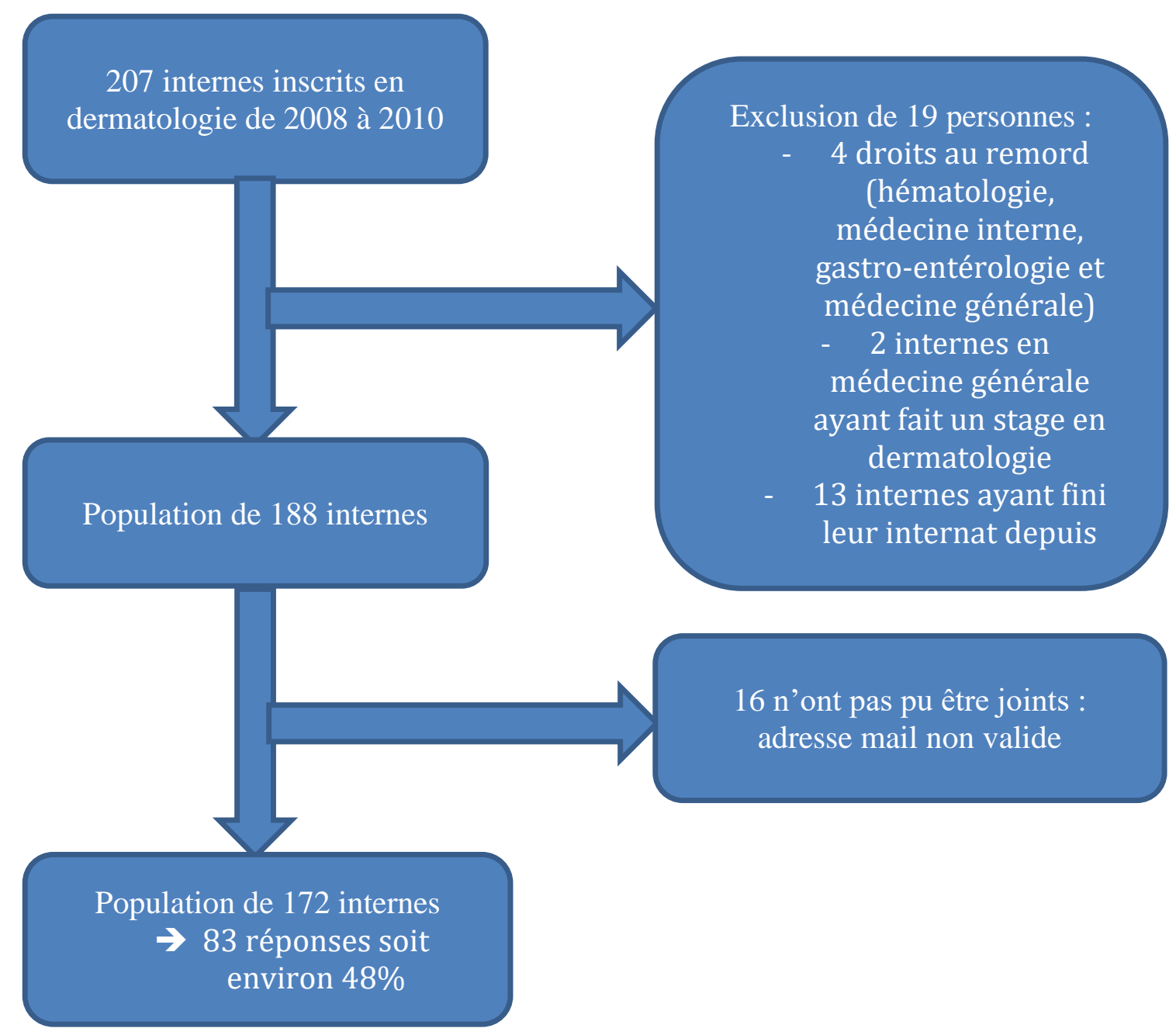

Figure 2. Flow chart.

Notre effectif comptait 77 femmes soit $93 \%$ et 6 hommes soit $7 \%$ âgés de 31 à 36 ans avec un âge moyen de 33,4 ans et une médiane de 33 ans.

Les effectifs des participants en fonction de leur CHU d'origine sont rapportés sur la figure 3. 


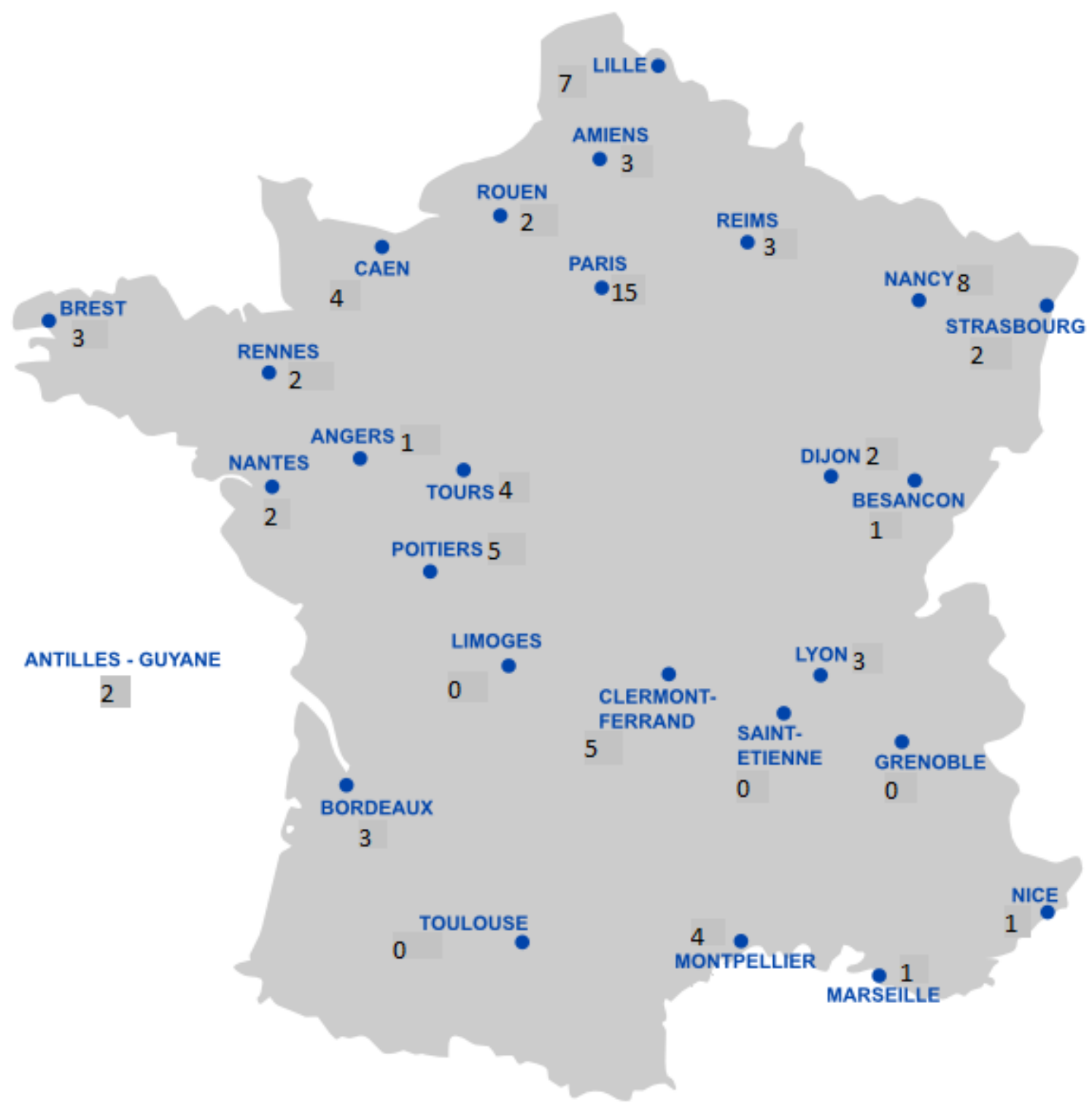

Figure 3. Effectifs de répondeurs par CHU d'origine.

Deux tiers de notre population est restée dans la région du CHU de formation. Parmi le tiers ayant migré,

- $52 \%$ avaient suivi leur conjoint

- $48 \%$ étaient retournés dans leur région d'origine

- $28 \%$ avaient eu une opportunité d'un poste ailleurs

- $24 \%$ avaient simplement eu envie de changement.

La majorité des jeunes dermatologues avait réalisé un clinicat (43\%), environ un tiers un assistanat (35\%) et environ un quart (22\%) n'avait pas fait de post-internat. 
Parmi ceux n'ayant pas fait de post-internat (22\% soit 18 jeunes dermatologues),

- 13 travaillaient en cabinet libéral $(72 \%)$ et donc en secteur I

- 3 avaient une activité mixte en cabinet/CH (Centre Hospitalier) périphérique (17\%)

- 1 travaillait en CH Périphérique (5.5\%)

- 1 travaillait en centre de santé $(5.5 \%)$

- Aucun en CHU.

Plus de la moitité (54\%) des jeunes dermatologues exerçaient toujours dans une ville de CHU et environ $20 \%$ dans une ville de moins de 30000 habitants. Seuls 17\% d'entre eux exerçaient dans une zone considérée comme désert médical.

Ils citaient dans les commentaires libres de la question 30 notamment : "surcharge de travail », « mauvaises conditions de travail » et « pas d'incitation à s'y installer ». Ils disaient ne plus prendre de nouveaux patients sauf sur demande explicite et insistante du médecin traitant avec des délais pouvant aller jusqu'à 2 ans, refusant plus de 25 patients/jour et étant obligé d'espacer les suivis carcinologiques à une durée de 18 mois pour des patients devant parfois être suivis tous les 6 mois. Ils travaillaient souvent en tant que seul dermatologue d'un large périmètre et rapportaient la notion de «surbooking ».

Concernant la structure d'excercice (graphique 1), 60\% exerçaient en cabinet libéral, 27\% en $\mathrm{CHU}, 27 \%$ en $\mathrm{CH}$ périphérique, $7 \%$ en centre de santé, $6 \%$ en clinique et $1 \%$ en unité INSERM (Institut National de la Santé et de la Recherche Médicale).

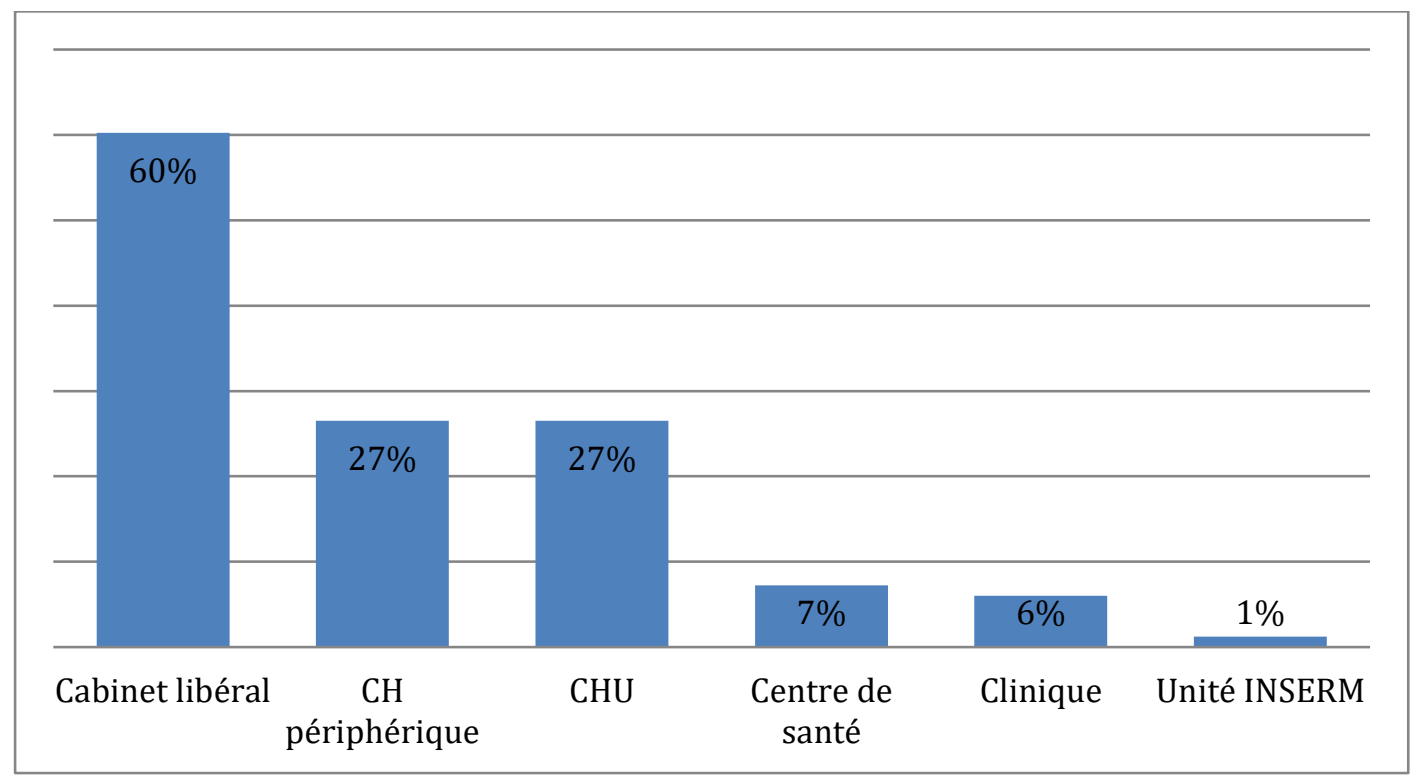

Graphique 1. Structure d'exercice. 
Concernant le statut d'exercice (graphique 2), parmi les $57 \%$ en activité libérale, 29\% présentaient 2 statuts concomitants.

En ce qui concerne l'activité hospitalière, on dénombrait 29\% de PH (Praticien Hospitalier), toutes des femmes : $19 \%$ à temps plein, $12 \%$ à temps partiel et $17 \%$ étaient vacataires. Parmi les non-répondants, 2 hommes étaient $\mathrm{PH}$, après recherche de leur statut sur internet.

Cinquante-six pourcents des $\mathrm{PH}$ ont fait des remplacements et $44 \%$ n'en ont jamais fait et 94\% des PH à temps plein ont fait un post-internat (50\% assistants et 50\% chef de clinique).

Parmi le reste de la population, 17\% faisaient encore des remplacements, $10 \%$ exerçaient dans un centre de santé et $2 \%$ étaient en cursus universitaires.

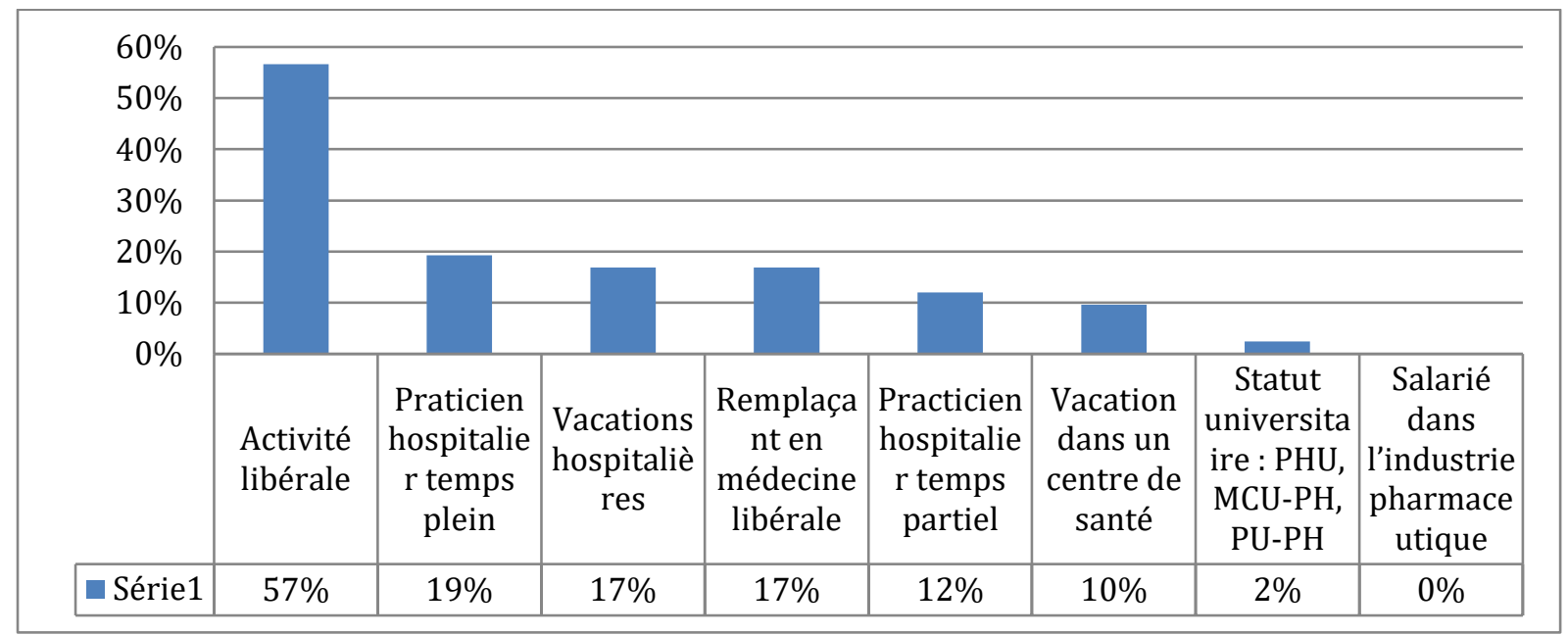

Graphique 2. Statut d'exercice.

Quatre-vingt six pourcents des jeunes dermatologues ont fait des remplacements dont la durée moyenne est de 2.2 ans avec un écart-type de 1,3 an et une médiane à 2 ans. Ces remplacements étaient réalisés :

- à temps partiel dans $74 \%$ des cas (dont $12 \%$ en statut d'auto-entrepreneur)

- à temps plein dans $26 \%$ des cas.

Pour $87 \%$ des répondants, la dermatologie générale correspondait à plus de $75 \%$ de leur temps d'activité et à plus de 50\% pour $93 \%$ d'entre eux. Seuls $7 \%$ faisaient plus de médecine esthétique que de dermatologie générale. La moyenne de demi-journées travaillées par semaine était de 8 avec un écart-type de 1,8 et une médiane de 8 . 
Dix huit pourcents des jeunes dermatologues ne faisaient pas de consultations spécialisées (graphique 3). Les principales sur-spécialisations des autres $81 \%$ étaient la cancérologie (35\%), le psoriasis $(27 \%)$, la dermatopédiatrie $(24 \%)$ et la médecine esthétique (18\%).

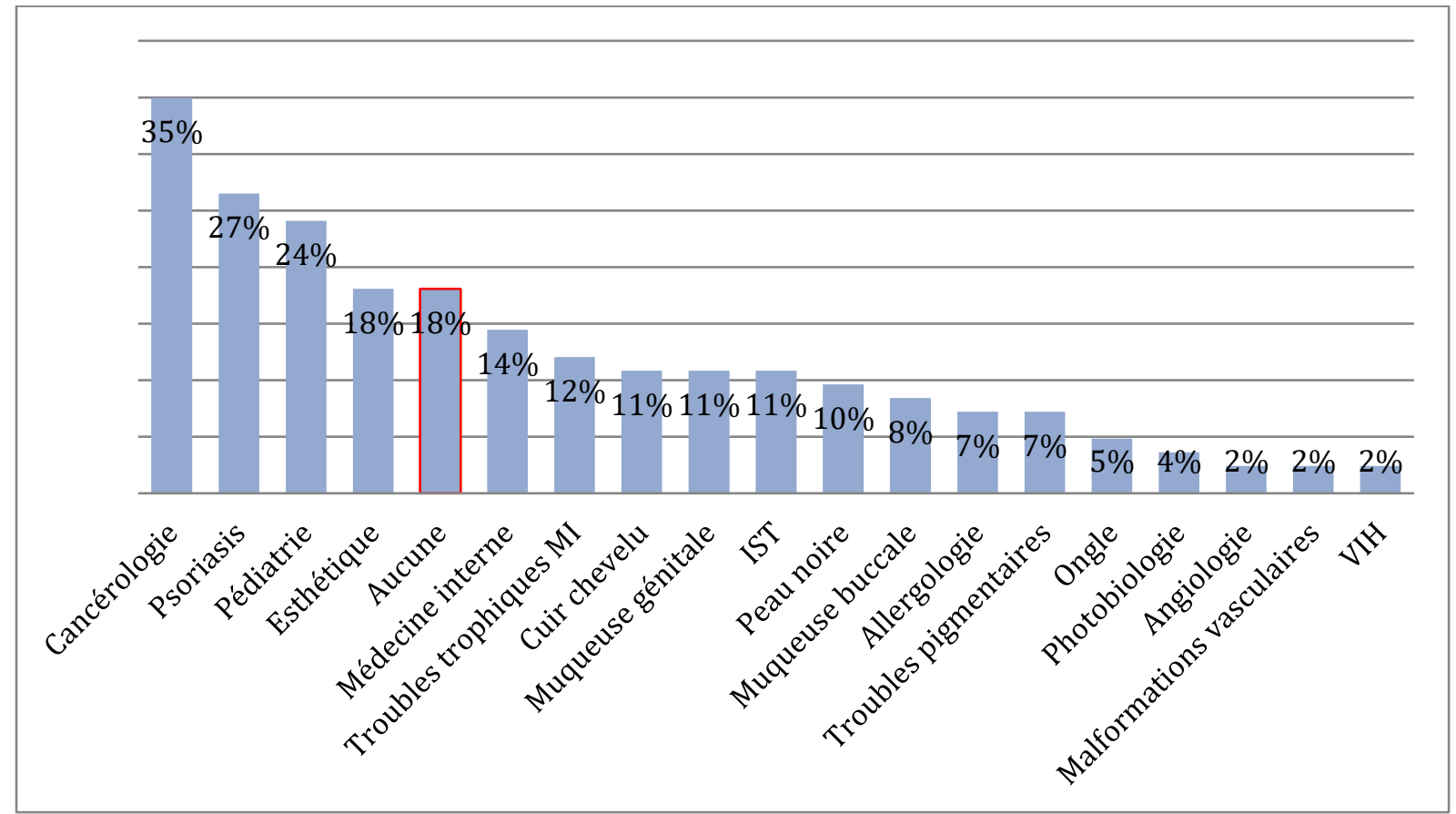

Graphique 3. Sur-spécialisations.

Ces consultations étaient dans la moitié des cas réalisées en milieu hospitalier et dans $45 \%$ des cas en cabinet libéral (graphique 4).

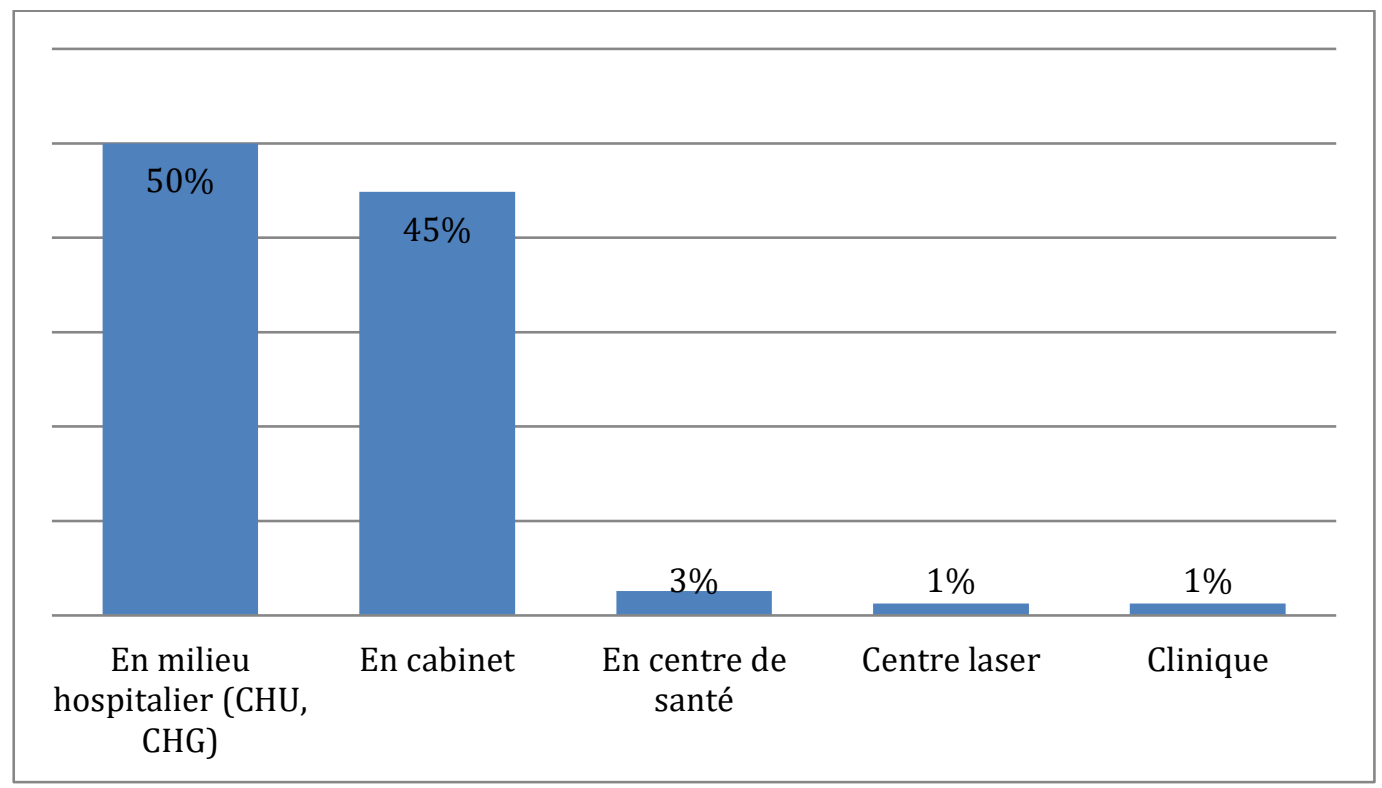

Graphique 4. Lieu de réalisation des consultations spécialisées. 
La quasi-totalité des jeunes dermatologues faisaient de la chirurgie dermatologique ; presque la moitié d'entre eux avaient accès à une cabine à UV, un appareil de photothérapie dynamique ou à un laser ; un quart faisaient de l'esthétique ; et un quart de la cryochirurgie (graphique 5).

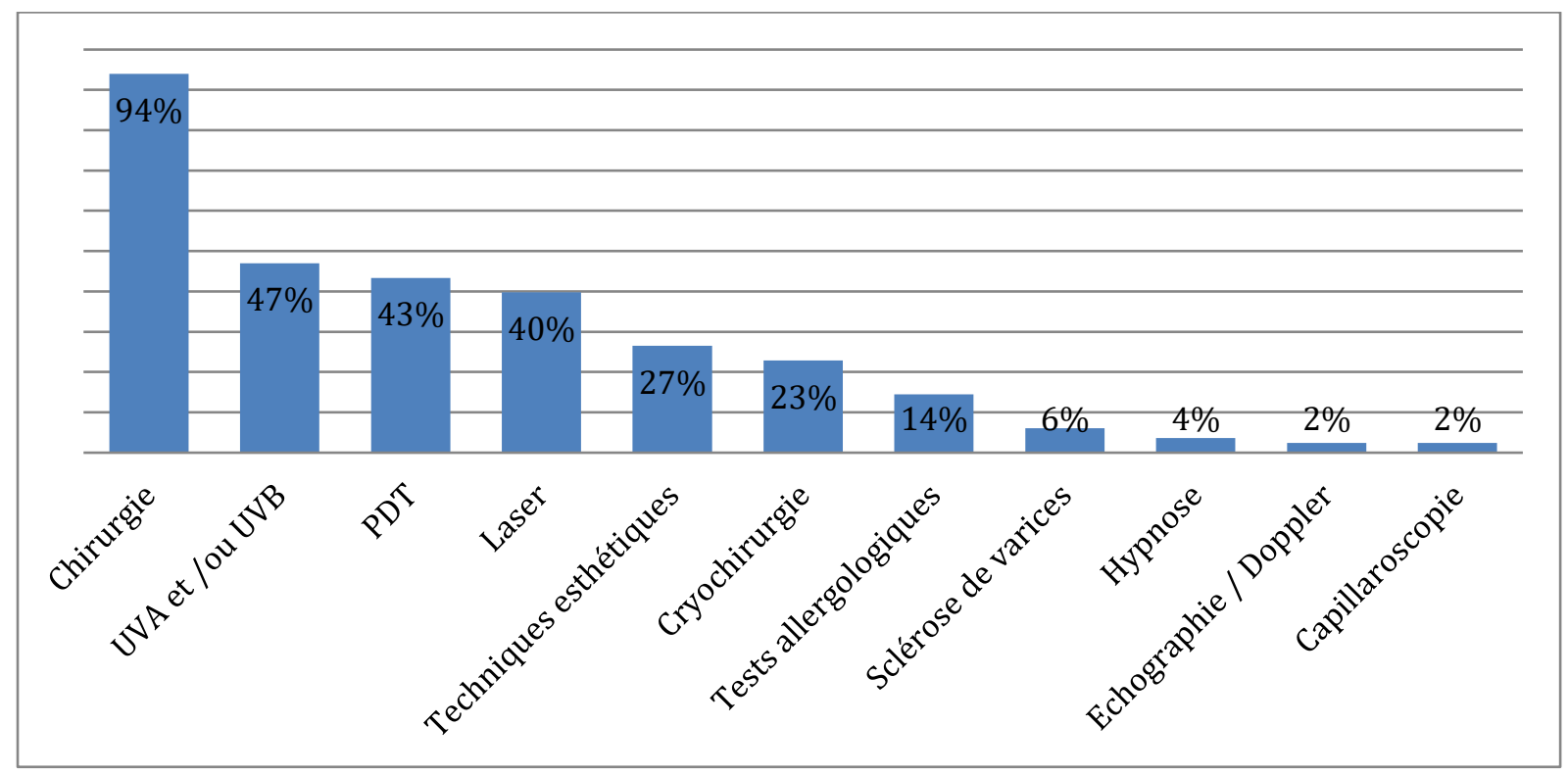

Graphique 5. Techniques pratiquées.

Parmi les $94 \%$ pratiquant la chirurgie dermatologique, $74 \%$ ne faisaient que de la «petite chirurgie » (biopsies et exérèses nécessitant une reconstruction par suture simple), 25\% pratiquaient les exérèses avec reconstruction par greffes cutanées ou lambeau et $1 \%$ opéraient sous anesthésie générale. L'activité chirurgicale occupait 1 à 3 demi-journées/semaine avec une moyenne de 1,36 un écart-type de 0,6 et une médiane de 1.

L'activité laser, pratiquée par $40 \%$ de notre population, était répartie sur 1 à 4 demijournées/semaine avec une moyenne de 1,2, un écart-type de 0,6 et une médiane de 1 . L'utilisation des lasers était principalement à visée dépilatoire et vasculaire (graphique 6). 


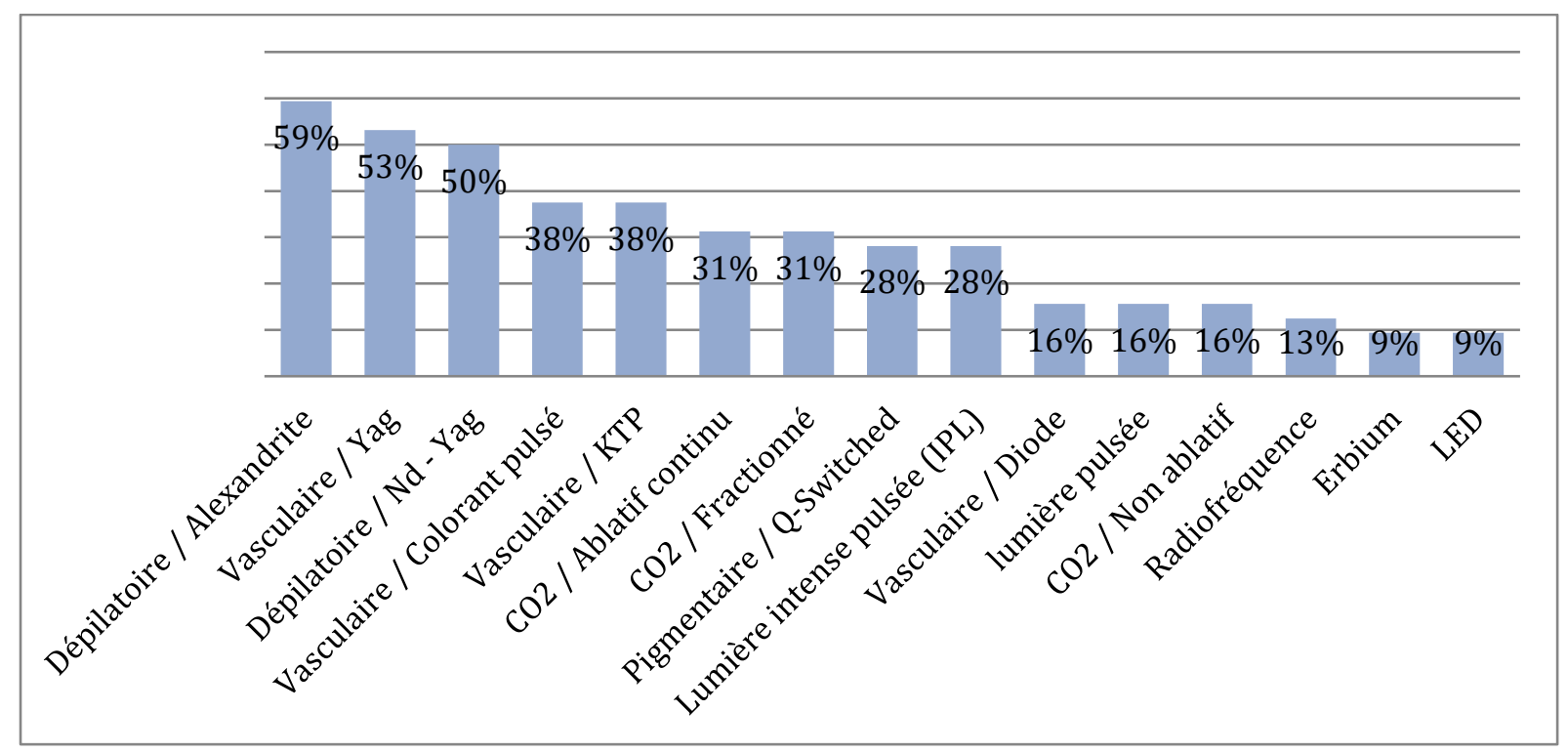

Graphique 6. Lasers utilisés.

En dehors du laser, l'activité esthétique était dominée par les peelings chimiques surtout superficiels puis les produits de comblement type acide hyaluronique suivis de près par la toxine botulique (graphique 7).

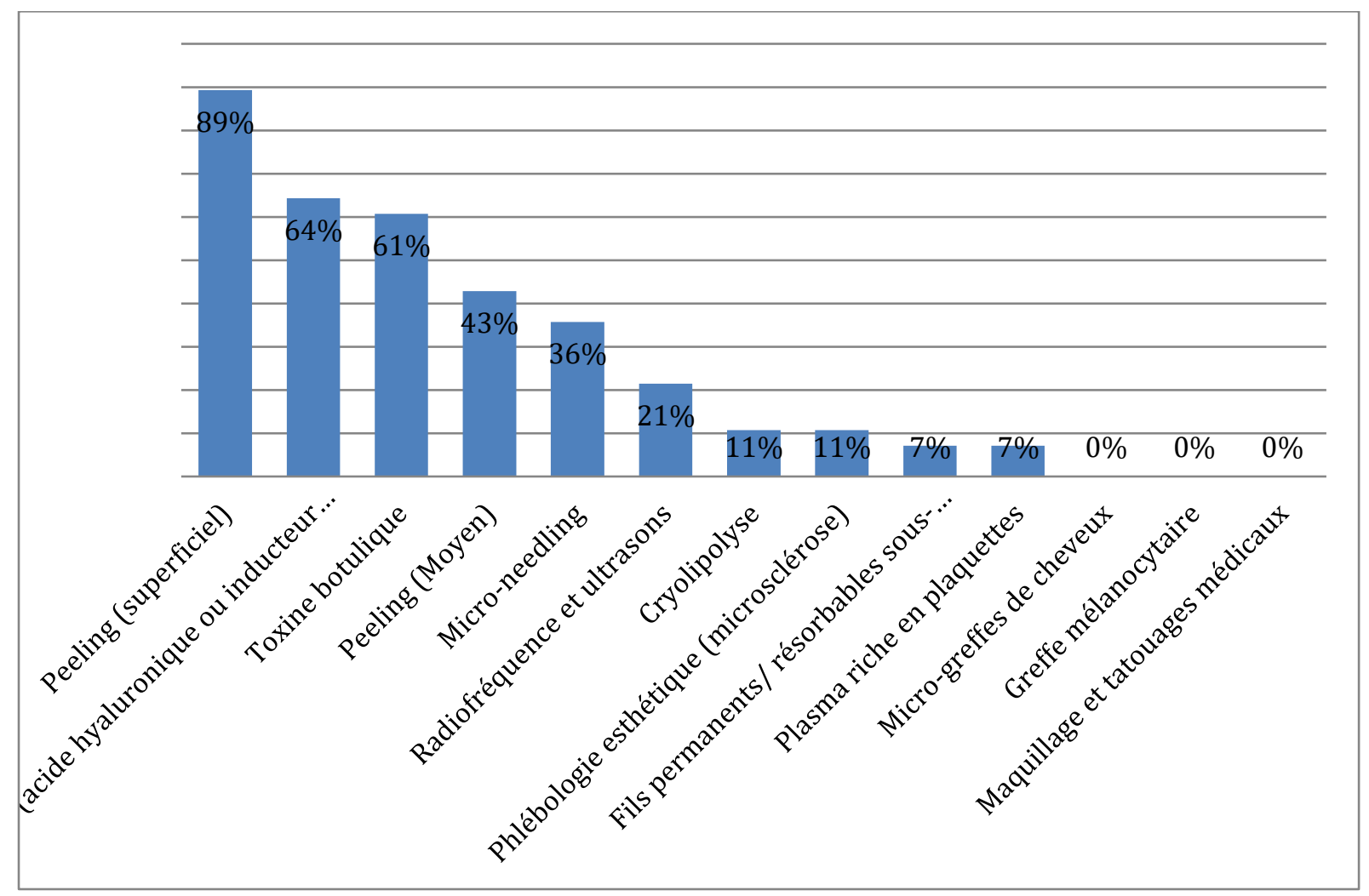

Graphique 7. Techniques esthétiques pratiquées. 
Soixante six pourcents des participants de notre étude déclaraient avoir une activité libérale :

- secteur d'activité :

- $57 \%$ étaient conventionnés en secteur 2

- $37 \%$ secteur 1

- $6 \%$ sont non conventionnés

- $\quad 60 \%$ en cabinet et $6 \%$ en clinique.

Dix huit pourcents n'étaient pas encore installés. Pour les autres, l'installation avait lieu en moyenne de 3,1 ans (écart-type de 1,6 et médiane de 3) après la fin de l'internat. Ce qui correspondait à presque 1 an de plus que la moyenne de remplacement avant installation de 2.2 ans retrouvés.

Parmi ceux s'étant installés seuls, on notait une proportion quasi-égale entre l'ouverture d'un nouveau cabinet $(15 \%)$ et la reprise du cabinet d'un dermatologue partant en retraite $(13 \%)$. Pour ceux installés en association avec d'autres dermatologues, la majorité (33\%) intégrait un cabinet et $13 \%$ en ouvraient un. Seuls 5\% s'installaient avec d'autres spécialistes/généralistes ou d'autres paramédicaux. A noter que $15 \%$ faisaient toujours des remplacements et $2 \%$ étaient partis travailler à l'étranger.

Ainsi, $28 \%$ avaient fait les démarches pour ouvrir une structure (15\% seuls et $13 \%$ en association) contre 53\% de notre population qui intégraient une structure déjà existante (33\% en association avec des dermatologues, $13 \%$ un cabinet laissé par un retraité, 3\% une maison médicale, $2 \%$ une maison de santé pluridisciplinaire et $2 \%$ un centre de laser (graphique 8).

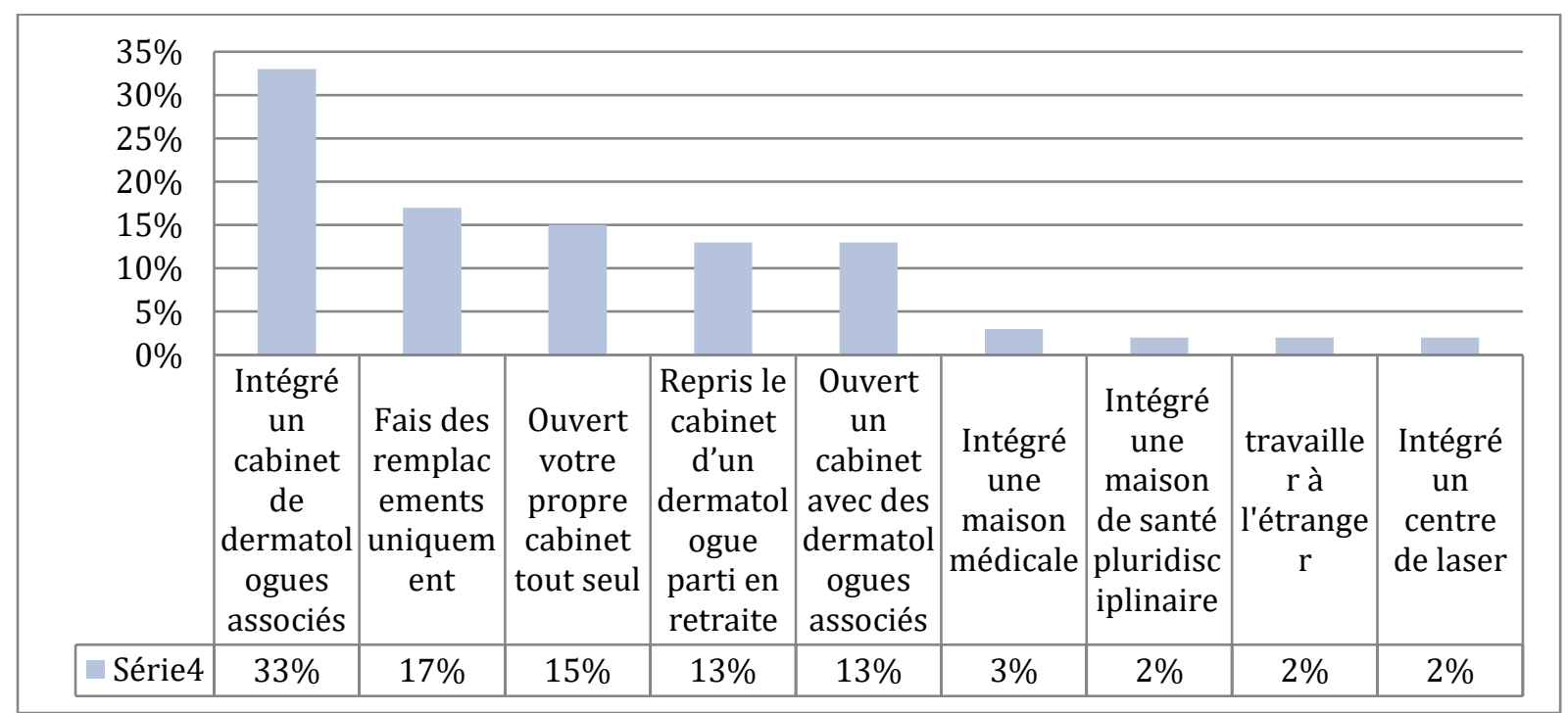

Graphique 8. Situation au démarrage de l'activité libérale. 
Vingt pourcents de la population installés en libéral déclaraient avoir eu des difficultés à s'installer pour diverses raisons (financières, modalités administratives, modalité de comptabilité, relation avec les confrères...etc).

Pour ceux qui n'étaient pas installés, les freins à l'installation étaient multiples :

- difficultés de gestion d'une entreprise pour $60 \%$;

- $\quad$ crainte des charges pour $54 \%$;

- pas envie pour $50 \%$;

- manque d'informations sur la pratique libérale pour 46\% ;

- manque de formation à la chambre de commerce dans $28 \%$;

- manque financier pour $13 \%$.

La moitié de notre population étudiée exerçaient déjà ou prévoyaient de pratiquer prochainement de la télémédecine avec une proportion équilibrée entre la téléconsultation et la téléexpertise en tant qu'expert :

- $22 \%$ de téléconsultation ;

- $21 \%$ de télé-expertise comme expert ;

- $7 \%$ de télé-expertise comme requérant.

Soixante et un pourcents des participants à l'étude ont souhaité recevoir les résultats et ont ainsi renoncé à l'anonymat en nous communicant leur adresse mail.

2. Comparaison de l'activité actuelle avec la formation reçue pendant l'internat

Pendant leur internat, les outils de formation auxquels ils ont eu recours en plus de leur cursus de DES (Diplôme d'Etudes Spécialisées) étaient principalement (graphique 9) :

- $\quad$ Les JDP $(95 \%)$

- Les DU (94\%)

- La participation à des FMC locales $(84 \%)$

- Et de la bibliographie, de la lecture (83\%). 


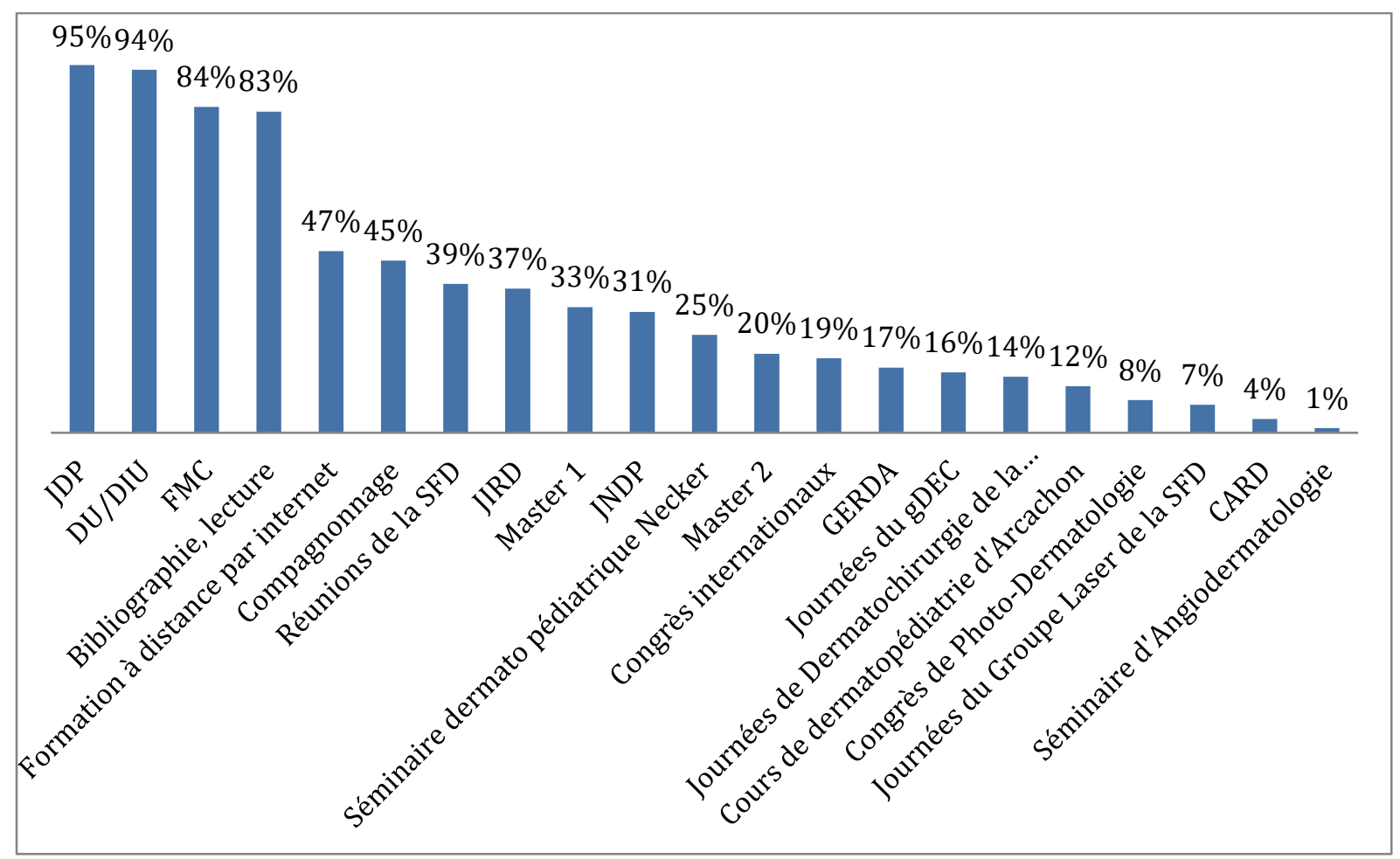

Graphique 9. Outils de formation pendant l'internat.

Et actuellement (graphique 10) :

- Bibliographie, lecture (70\%)

- $\operatorname{JDP}(65 \%)$

- Participation à des FMC locales/DPC (60\%)

- $\mathrm{DU}(53 \%)$.

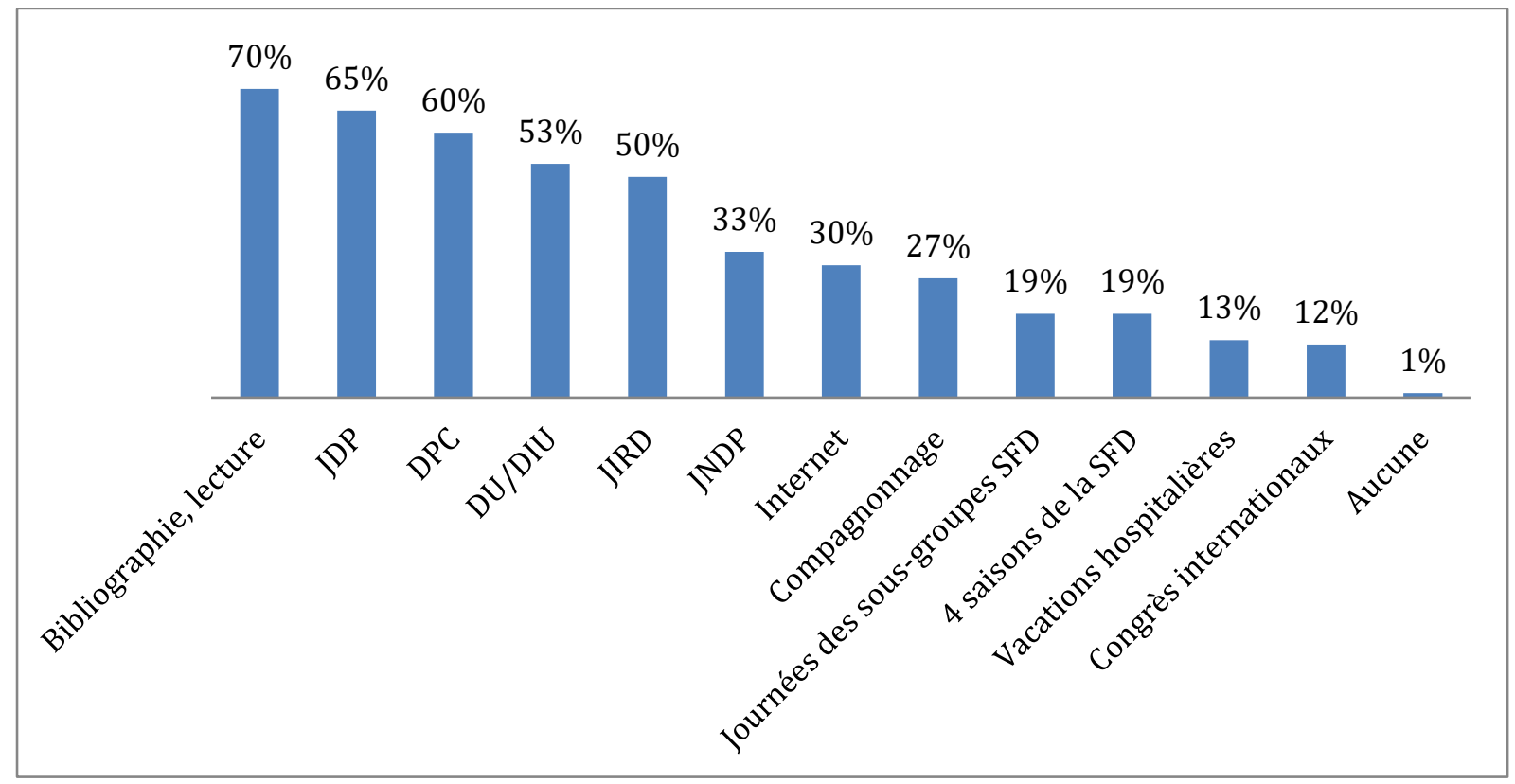

Graphique 10. Outils de formation actuels. 
La comparaison des outils de formation des internes en dermatologie et celles des jeunes dermatologues de notre population est rapportée dans le tableau 2.

\begin{tabular}{|c|c|c|}
\hline & Pendant l'internat & Actuellement \\
\hline DU & $94 \%$ & $53 \%$ \\
\hline Master I & $33 \%$ & $\mathbf{X}$ \\
\hline Master II & $20 \%$ & $\mathbf{X}$ \\
\hline FMC/DPC & $84 \%$ & $60 \%$ \\
\hline Réunions de la SFD & $39 \%$ & $19 \%$ \\
\hline JDP & $95 \%$ & $65 \%$ \\
\hline JNDP & $31 \%$ & $33 \%$ \\
\hline JIRD & $37 \%$ & $50 \%$ \\
\hline $\begin{array}{l}\text { Cours de dermatologie } \\
\text { pédiatrique d'Arcachon }\end{array}$ & $12 \%$ & $X$ \\
\hline $\begin{array}{c}\text { Séminaire de dermatologie } \\
\text { pédiatrique de Necker }\end{array}$ & $25 \%$ & Uniquement pour les internes \\
\hline Sous-groupes de la SFD & $21 \%$ & $19 \%$ \\
\hline $\begin{array}{c}\text { Séminaire } \\
\text { d'angiodermatologie }\end{array}$ & $1 \%$ & N'existe plus \\
\hline $\begin{array}{c}\text { Congrès de } \\
\text { photodermatologie }\end{array}$ & $8 \%$ & N'existe plus \\
\hline CARD & $4 \%$ & N'existe plus \\
\hline Congrès internationaux & $19 \%$ & $12 \%$ \\
\hline Compagnonnage & $45 \%$ & $27 \%$ \\
\hline À distance par internet & $47 \%$ & $30 \%$ \\
\hline Bibliographie, lecture & $83 \%$ & $70 \%$ \\
\hline
\end{tabular}

Tableau 2. Comparaison des outils de formation pendant et après l'internat.

Seize pourcents se sont formés à l'esthétique en assistant aux journées annuelles du gDEC. Dix-huit pourcents de notre population déclaraient une activité esthétique et pour 7\%, cette pratique représentait plus de $50 \%$ de leur activité. 
L'activité laser se développe après l'internat avec $40 \%$ qui en pratiquaient contre seulement $7 \%$ qui assistaient aux journées de formation/congrès pendant l'internat.

La comparaison de l'enseignement reçu pendant l'internat et de l'activité actuelle des jeunes dermatologues de notre population est rapportée dans le tableau 3.

\begin{tabular}{|c|c|c|}
\hline & $\begin{array}{c}\text { Enseignement reçu ou en tout } \\
\text { cas proposé pendant } \\
\text { l'internat }\end{array}$ & Activité actuelle \\
\hline $\begin{array}{l}\text { Dermatologie esthétique et } \\
\text { correctrice }\end{array}$ & $\begin{array}{c}16 \% \text { ont assisté aux journées } \\
\text { de formation }\end{array}$ & $\begin{array}{c}18 \% \text { et } 7 \% \text { en font plus de } \\
50 \% \text { de leur activité }\end{array}$ \\
\hline Dermatologie pédiatrique & $\begin{array}{l}\text { Un séminaire à 1'hôpital } \\
\text { Necker (25\%) } \\
\text { Cours d'Arcachon (12\%) }\end{array}$ & $\begin{array}{l}24 \% \text { se disent spécialisés en } \\
\text { dermatopédiatrie }\end{array}$ \\
\hline $\begin{array}{c}\text { Allergologie, congrès du } \\
\text { GERDA }\end{array}$ & $17 \%$ y ont assisté & $\begin{array}{l}7 \% \text { se disent spécialisés en } \\
\text { allergologie et } 14 \% \text { font des } \\
\text { tests allergologiques }\end{array}$ \\
\hline Angio-dermatologie & $\begin{array}{c}1 \text { séminaire réalisé par } 1 \% \text { de } \\
\text { la population }\end{array}$ & $\begin{array}{l}2 \% \text { en font encore } \\
\text { (capillaroscopie, doppler, } \\
\text { sclérose de varices) }\end{array}$ \\
\hline $\begin{array}{c}\text { Journées du groupe laser de } \\
\text { la SFD }\end{array}$ & $7 \%$ y ont assisté/participé & $40 \%$ font du laser \\
\hline $\begin{array}{c}\text { Congrès de la société } \\
\text { française de } \\
\text { photodermatologie }\end{array}$ & $8 \%$ y ont assisté & $\begin{array}{c}4 \% \text { font de la } \\
\text { photodermatologie }\end{array}$ \\
\hline
\end{tabular}

Tableau 3. Comparaison de l'enseignement reçu avec l'activité actuelle.

3. Comparaison avec la population de tous les dermatologues français

Les jeunes dermatologues exerçant depuis moins de 10 ans représentaient environ $15 \%$ des dermatologues français (graphique 11). Notre population (ayant fini depuis 5 à 7 ans) correspondait à $2,7 \%$. 


\section{Proportion (\%)}

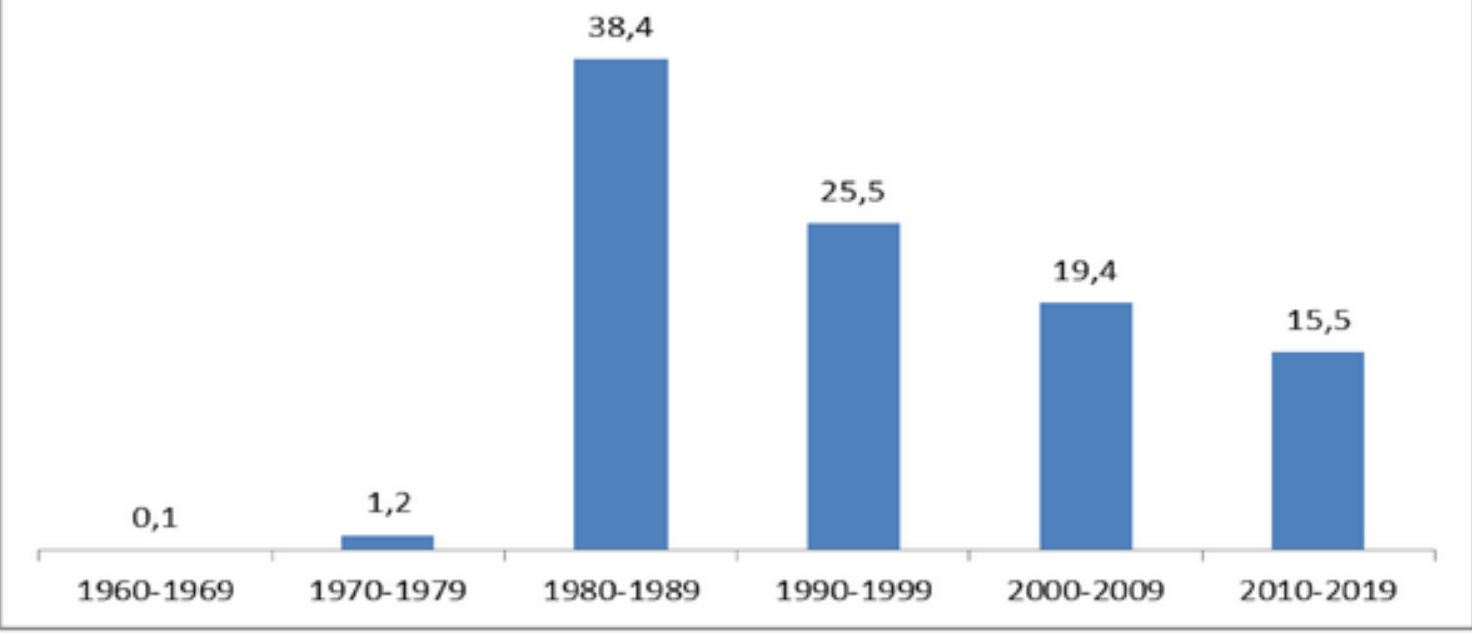

Graphique 11. Proportion de dermatologues classés par l'année de début d'activité (1).

Les questions d'une enquête similaire à la nôtre a été réalisée par la FFFCEDV ((Fédération Française de Formation Continue et d'Evaluation en Dermatologie Vénéréologie) en fin 2018, portaient aussi sur les caractéristiques de cette population, le mode et statut d'exercice, l'organisation du temps de travail et la pratique libérale.

L'étude concernait tous les dermatologues de France, soit environ 3000 et 899 réponses (30\%) ont été obtenues.

Le taux de femmes était nettement supérieur dans notre population de jeunes dermatologues (93\%) par rapport à celle de tous les dermatologues (75\%). Il en était de même pour l'activité en secteur II qui était majoritaire à 57\% dans notre population alors que c'était l'activité en secteur I qui était prépondérante dans la population globale des dermatologues avec $55 \%$.

Dans notre population, seuls $28 \%$ des libéraux exerçaient seul, $15 \%$ remplaçaient encore et $51 \%$ étaient installés en association, majoritairement avec d'autres dermatologues (46\%). $52 \%$ de tous les dermatologues exerçaient seuls et parmi les autres, 35\% installés avec d'autres dermatologues et $13 \%$ avec d'autres spécialistes.

La comparaison des caractéristiques des 2 populations est rapportée dans le tableau 4. 


\begin{tabular}{|c|c|c|}
\hline & Notre population & Tous dermatologues de France \\
\hline $\begin{array}{c}\text { Taux de } \\
\text { participation }\end{array}$ & $83 / 172=48 \%$ & $899 / 3000=30 \%$ \\
\hline Sexe & $\begin{array}{l}93 \% \text { de femmes } \\
7 \% \text { d'hommes }\end{array}$ & $\begin{array}{l}75 \% \text { de femmes } \\
25 \% \text { d'hommes }\end{array}$ \\
\hline Age moyen & 33,4 ans (31-36 ans) & 55 ans (29-79 ans) \\
\hline Secteur d'activité & $\begin{array}{c}\text { I : } 37 \% \\
\text { II }: 57 \% \\
\text { Non conventionné }: 6 \%=3 \\
\text { personnes }\end{array}$ & $\begin{array}{c}\text { I : } 55 \% \\
\text { II : } 45 \% \\
\text { Non conventionné }: 0,2 \%=2 \text { personnes }\end{array}$ \\
\hline $\begin{array}{c}\text { Mode d'exercice en } \\
\text { libéral }\end{array}$ & $\begin{array}{c}\text { Seul : } 28 \% \\
\text { Avec d'autres spécialistes : } 5 \% \\
\text { Avec d'autres dermatologues : } 46 \% \\
\text { Remplacements : } 17 \%\end{array}$ & $\begin{array}{c}\text { Seul : } 52 \% \\
\text { Avec d'autres spécialistes : } 13 \% \\
\text { Avec d'autres dermatologues : } 35 \%\end{array}$ \\
\hline Hospitalier & $\begin{array}{l}48 \% \text { sont hospitaliers : } \\
\text { - } 19 \% \text { à temps plein } \\
\text { - } 17 \% \text { font des vacations } \\
\text { - } 12 \% \text { à temps partiel } \\
\text { - } 27 \% \text { travaillent en CHU et } \\
27 \% \text { en } \mathrm{CH} \text { périphérique }\end{array}$ & $\begin{array}{l}32 \% \text { sont hospitaliers : } \\
\text { - } 72 \% \text { vacataires } \\
\text { - } 17 \% \text { à temps plein } \\
\text { - } 11 \% \text { à temps partiel }\end{array}$ \\
\hline Remplacements & $\begin{array}{l}86 \% \text { ont fait des remplacements } \\
\text { dont } 26 \% \text { à temps plein, en } \\
\text { moyenne } 2,8 \text { ans avant installation, } \\
17 \% \text { en faisaient encore } \\
\text { actuellement }\end{array}$ & $\begin{array}{l}26 \% \text { se font remplacés et } 4 \% \text { font des } \\
\text { remplacements à une moyenne de } 3 \\
\text { jours/semaine }\end{array}$ \\
\hline Formations actuelles & $\begin{array}{c}\text { DPC/FMC : } 60 \% \\
\text { SFD : } 19 \% \\
\text { Sous-groupes SFD : } 19 \%\end{array}$ & $\begin{array}{c}\text { DPC/FMC : } 65 \% \\
\text { FFCEDV : } 77 \% \\
\text { SFD : } 57 \% \\
\text { SNDV : } 43 \% \\
\text { Sous-groupes SFD : } 25 \%\end{array}$ \\
\hline Congrès & $\begin{array}{l}\text { JDP : } 65 \% \\
\text { JIRD : } 50 \%\end{array}$ & $\begin{array}{l}\text { JDP }: 536 / 899=60 \% \\
\text { JIRD : } 445 / 899=49 \%\end{array}$ \\
\hline Formation en ligne & $30 \%$ & $491 / 899=55 \%$ \\
\hline
\end{tabular}

Tableau 4. Caractéristiques des 2 populations. 
La grande majorité des médecins toutes spécialités confondues nouvellement inscrits au CNOM (Conseil National de l'Ordre des Médecins) a un exercice salarié majoritaire. Les dermatologues inscrits au CNOM choisissent d'exercer pour $58 \%$ en secteur libéral, $22 \%$ en tant que salariés (20\% exercice mixte) (1). Dans notre population, les chiffres sont un peu différents avec $42 \%$ d'activité libérale, $29 \%$ de salariés et $29 \%$ d'activité mixte.

La répartition de l'activité de tous les dermatologues en France en janvier 2018 d'après le CNOM (1) et celle des jeunes dermatologues de notre population questionnés en janvier 2019 sont rapportées respectivement dans les tableaux 5 et 6 .

\begin{tabular}{|c|c|c|c|c|}
\hline & \multicolumn{2}{|c|}{ Libéral } & Mixte & Salarié \\
\hline Hommes & \multicolumn{2}{|c|}{529} & 255 & 165 \\
\hline Femmes & \multicolumn{2}{|c|}{1408} & 395 & 576 \\
\hline & Libéral & Mixte & Salarié & Total \\
\hline Hommes & $56 \%$ & $27 \%$ & $17 \%$ & $29 \%$ \\
\hline Femme & $59 \%$ & $17 \%$ & $24 \%$ & $71 \%$ \\
\hline Total & $58 \%$ & $20 \%$ & $22 \%$ & $100 \%$ \\
\hline
\end{tabular}

Tableau 5. Activité de tous les dermatologues de France (1).

\begin{tabular}{|c|c|c|c|c|}
\hline & Libéral & Mixte & Salarié & Total \\
\hline \multirow{2}{*}{$\begin{array}{l}\text { Hommes } \\
\text { Femmes }\end{array}$} & 2 & 3 & 1 & 6 \\
\cline { 2 - 5 } & 33 & 21 & 23 & 77 \\
\hline
\end{tabular}

\begin{tabular}{|c|c|c|c|c|}
\hline & Libéral & Mixte & Salarié & Total \\
\hline Hommes & $33 \%$ & $50 \%$ & $17 \%$ & $7 \%$ \\
\hline Femmes & $43 \%$ & $27 \%$ & $30 \%$ & $93 \%$ \\
\hline Total & $42 \%$ & $29 \%$ & $29 \%$ & $100 \%$ \\
\hline
\end{tabular}

Tableau 6. Activité des dermatologues de notre population.

\section{DISCUSSION}

Le nombre de dermatologues en activité diminue chaque année de 3821 en janvier 2007 jusqu'à avoir atteint 3328 en janvier 2018. Par comparaison, la population des cardiologues 
est passée de 5966 en 2007 à 6246 en 2018 et celle des pédiatres de 6464 en 2007 à 7334 en $2018(1,4)$.

Dans les années 1990, à la suite de décisions politiques, le nombre d'étudiants autorisés à s'inscrire en dermatologie a été considérable réduit. Si bien qu'aujourd'hui, les nouveaux dermatologues ne compensent pas le nombre de départs en retraite, créant ainsi une pénurie de dermatologues en France (5).

En réaction à cela, le ministère des affaires sociales, de la santé et des droits des femmes a proposé d'augmenter le nombre de postes proposés en dermatologie aux ECN. En 2010, il y avait 73 postes en dermatologie à l'ECN, il y en a 91 en 2019. Le numérus clausus a aussi été doublé ces 10 dernières années. À terme, cette augmentation devrait profiter à l'ensemble des spécialités médicales dont la dermatologie (6).

\section{L'atlas démographique de 2018 du CNOM démontre une population médicale} vieillissante (graphiques 12 et 13): seulement $15 \%$ des dermatologues et $18 \%$ de tous médecins ont moins de 40 ans ; et $34 \%$ des dermatologues et $45 \%$ de tous médecins ont plus de 60 ans (graphiques 12 et 13) (1). Celui de 2015 faisait mention que chaque année, pas moins de $25 \%$ des médecins nouvellement diplômés d'une faculté française décident de ne pas s'inscrire à l'Ordre pour exercer d'autres professions : journalisme, administration etc. (2).

Le nombre de dermatologues inscrits au tableau de l'Ordre augmente de 1 à $2 \%$ chaque année mais majoritairement au bénéfice de médecins retraités qui poursuivent leur activité. Ces derniers correspondent à 5,9\% des médecins en activité en janvier 2018 (1).

La dermatologie est une spécialité qui se féminise avec le temps, non pas par une augmentation du nombre de femmes mais plutôt par une diminution du nombre d'hommes (graphique 12). 


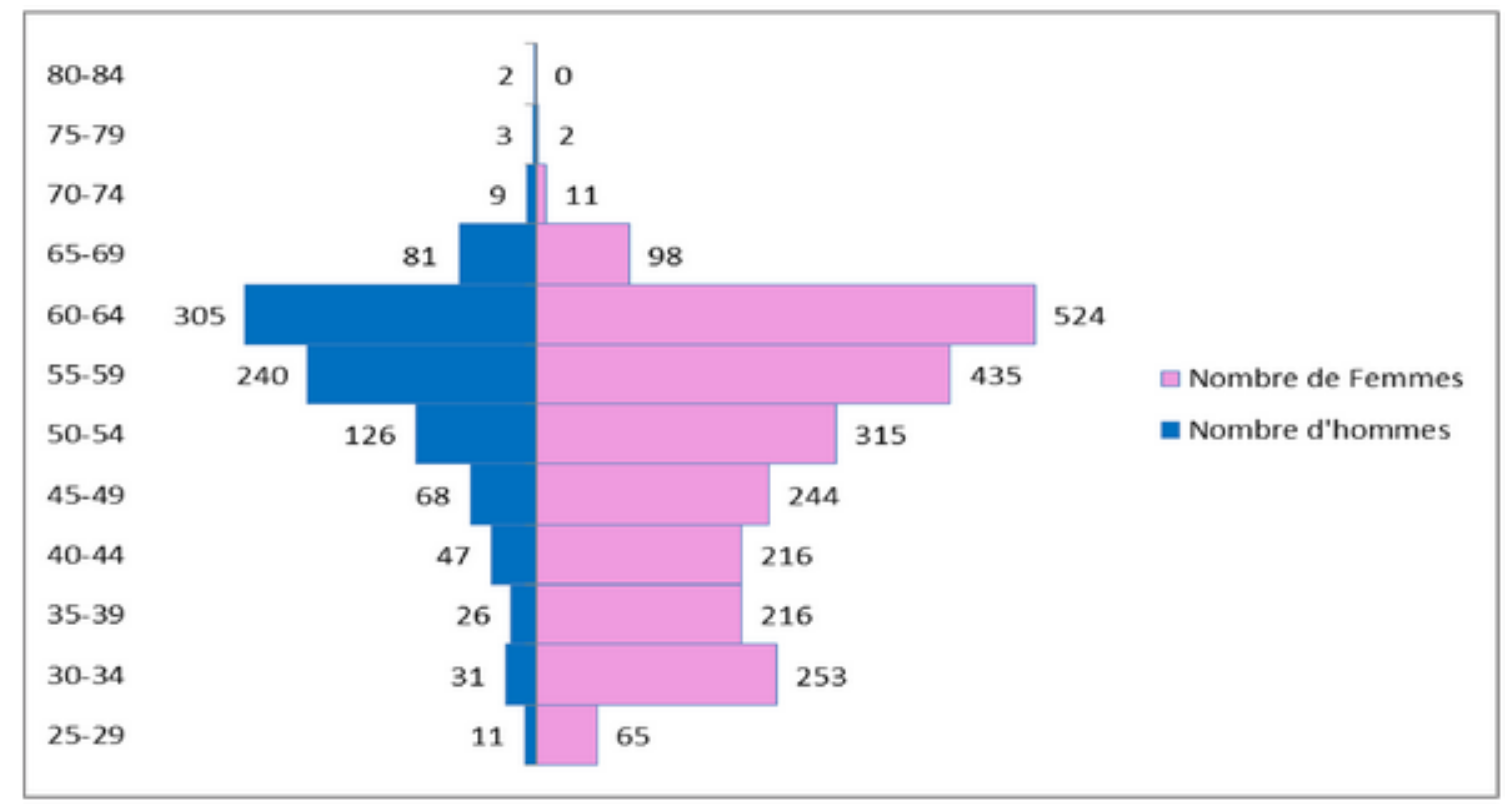

Graphique 12. Pyramide des âges en dermatologie en janvier 2018 (1).

Sur la tranche d'âge de 30 à 34 ans du graphique 12 qui correspond à notre population d'étude, on retrouve $89 \%$ de femmes (253) et $11 \%$ d'hommes (31). Parmi les répondeurs à notre étude, il y avait $93 \%$ de femmes et $7 \%$ d'hommes et parmi les gens n'ayant pas pu être joint, il y avait $24 \%$ d'hommes et $76 \%$ de femmes. Ainsi, sur l'effectif total des dermatologues ayant fini depuis 5 à 7 ans, soit les 188 personnes initiales, on retrouve bien $89 \%$ de femmes et $11 \%$ d'hommes.

Il existe un parallèle avec la pyramide des âges de tous les médecins en activité régulière en 2018 (graphique 13).

La dermatologie n'est pas l'exception en termes de féminisation. En effet, selon l'atlas de la démographie médicale de janvier 2018 du CNOM, les femmes médecins sont beaucoup plus nombreuses que les hommes chez les moins de 45 ans et 59\% des nouveaux inscrits à l'ordre sont des femmes. On observe d'ailleurs un lien inversement proportionnel entre l'âge moyen des médecins actifs et le taux de féminisation des médecins, montrant bien la forte féminisation des études médicales récente sur les dernières décennies (graphique 13). 


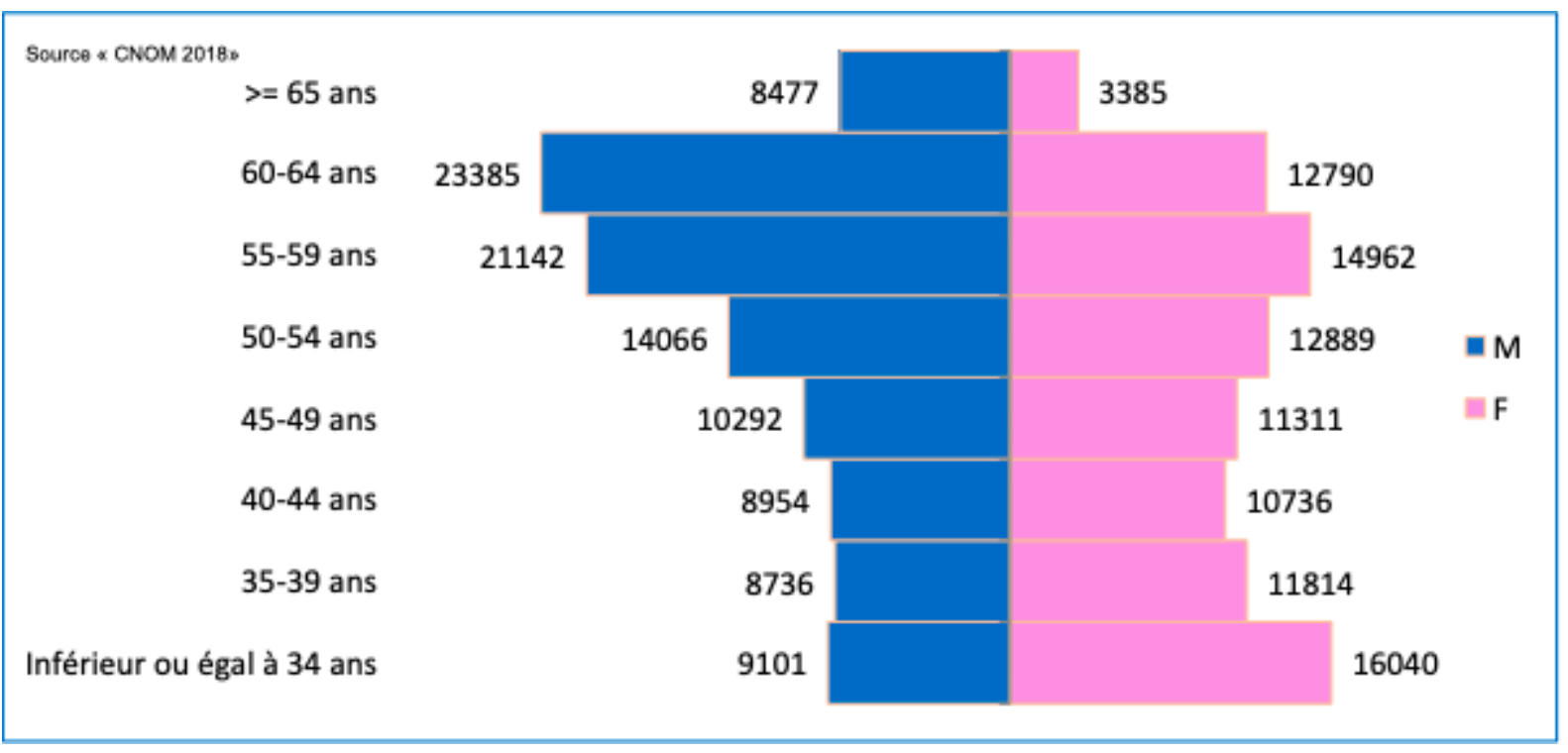

Graphique 13. Pyramide des âges des médecins en activité régulière en 2018 (1).

La féminisation du corps médical constitue un phénomène présent dans quasiment tous les pays occidentaux (7). Pour la France, cela a débuté à partir des année 70 sous l'influence directe de l'accès croissant des femmes aux études supérieures (8).

La moyenne de demi-journées travaillées par semaine de notre population était de 8 . Ce qui était déjà le cas en 2004 pour les dermatologues libéraux (9). Les charges domestiques et éducatives des femmes semblent encore déterminer leurs modes d'exercice. Certains sociologues y voient un risque pour l'avenir de la profession médicale par remise en question du principe de la «disponibilité permanente» des médecins et ainsi une dévalorisation du statut de médecin (10).

La quasi-totalité des PH (94\%) ont fait un post-internat. Le clinicat/assistanat représente des années de formation en plus de l'internat toujours dans un encadrement universitaire. Lorsque l'on se tourne vers une activité hospitalière, il est plus intéressant de faire un postinternat. Par ailleurs, pour passer le concours de $\mathrm{PH}$, il peut être indispensable d'avoir été chef de clinique ou assistant, surtout dans un CHU.

D'ailleurs, parmi ceux n'ayant pas fait de post-internat, seul 1/18 est $\mathrm{PH}$ en $\mathrm{CH}$ périphérique et $89 \%$ sont en libéral. 
La part d'activité libérale exclusive diminue de $58 \%$ de tous les dermatologues à $42 \%$ des jeunes dermatologues. D'après l'atlas de la démographie médicale en France de 2015 du CNOM, la dermatologie fait partie des 4 spécialités en souffrance (avec la rhumatologie, la chirurgie générale et l'ORL) concernant le mode d'exercice en libéral avec une baisse de $7,3 \%$ de 2007 à 2015 (2).

La différence est plus frappante chez les hommes qui passent de 56\% d'exercice libéral et $27 \%$ d'activité mixte parmi tous les dermatologues hommes de France à 50\% d'activité mixte et seulement 33\% d'activité libérale exclusive chez les jeunes hommes dermatologues.

Les femmes aussi délaissent peu à peu l'activité libérale (chute de 59\% à 43\%) pour se tourner vers l'exercice mixte (de $17 \%$ à $27 \%$ ) et le salariat (de $24 \%$ à $30 \%$ ).

Le Livre vert de la Commission relatif au personnel de santé en Europe précise la nécessité de donner une formation en gestion d'entreprise aux professionnels de la santé et de prendre en compte leur dimension de chef manager de leur cabinet $(11,12)$. Cependant, $60 \%$ de notre population ne s'installaient pas en libéral pour difficultés de gestion d'une entreprise, 46\% pour manque d'information et $28 \%$ pour manque de formation à la pratique libérale.

De plus, l'apprentissage de la spécialité se fait en équipe au sein d'un $\mathrm{CHU}$ avec que très peu de possibilité de stage en libéral.

\section{Ce mode d'exercice des jeunes dermatologues suit l'évolution des autres spécialités en}

France. Selon les données fournies par le CNOM dans son atlas démographique de 2015 : lors de leur première inscription à l'Ordre, $15 \%$ des jeunes médecins choisissent l'exercice libéral/mixte en 1ère intention, mais cinq ans plus tard, ils sont $40 \%$ à s'orienter vers ce mode d'exercice (2).

Si l'on considère tous les médecins français, l'atlas de démographie de 2018 rapporte une augmentation de presque $10 \%$ de l'effectif des salariés depuis 2010 (1).

\section{Le nombre de médecins titulaires d'un diplôme obtenu hors de France a augmenté} d'environ 43\% de 2007 et 2015. Cependant, ces médecins ne pallient pas au manque d'effectif car ils privilégient massivement l'exercice salarié $(62 \%)$ ou mixte $(13 \%)$. Seuls $25 \%$ d'entre eux exercent en secteur libéral exclusif (2). Les chiffres concernant les dermatologues titulaires d'un diplôme étranger exerçant en France n'ont pas été retrouvé. 


\section{La majorité des jeunes dermatologues exercent dans une ville de CHU et peu dans une}

zone considérée comme un désert médical est cohérent avec la carte démographique du CNOM (Page 10).

Il s'agit par ailleurs souvent de dermatologues seuls avec le confrère le plus proche pouvant être à $150 \mathrm{~km}$ de distance. De plus, le nombre grandissant de dermatologues partant en retraite et non remplacés aggravent la situation. Toutes ces raisons dissuadent beaucoup de jeunes dermatologues de venir s'installer dans ces zones de désert médical.

Les $17 \%$ installés en désert médical évoquaient des difficultés d'ordre professionnel et non personnel. Pourtant, un désert médical présente une attractivité et une qualité de vie réduite par la pauvreté des services à proximité (écoles, emploi pour le conjoint, loisirs) (11). La formation des internes se faisant en $\mathrm{CHU}$, les médecins sont ainsi conditionnés à travailler entourés et à vivre en zone urbaine.

Par ailleurs, les médecins titulaires d'un diplôme obtenu hors de France privilégient les territoires à forte densité et ne constituent pas réellement une réponse à la désertification (2).

L'augmentation du pourcentage de dermatologues exerçant en secteur II, corrélé à l'augmentation de la réalisation d'un post-internat (assistanat, clinicat) démontre la recherche d'une rentabilité financière supplémentaire des jeunes dermatologues. Le secteur I était prépondérant dans la population de tous les dermatologues de France.

\section{Cinquante deux pourcents de tous les dermatologues français installés en libéral sont} installés seuls contre seulement $\mathbf{2 8 \%}$ des jeunes de notre population. Ils sont installés avec d'autres dermatologues plutôt qu'avec d'autres spécialistes et préférentiellement dans une structure déjà existante. Cette volonté d'exercer en groupe n'est pas spécifique de la dermatologie. Le taux de médecins libéraux, toutes spécialités confondues, exerçant en groupe est en augmentation constante depuis plus d'une quarantaine d'années. Il est passé de $11 \%$ en 1969, à 27,4\% en 1982 pour atteindre plus de $44 \%$ en 2002. Ce regroupement reste principalement monodisciplinaire et de petite taille (2-3 médecins) (13). Il apparaît que l'une des motivations principales du regroupement porte sur le partage des moyens logistiques : partage des locaux de l'équipement et du personnel. Mais également en terme d'équilibre entre vie professionnelle et vie personnelle par partage des contraintes en terme de continuités des soins et de possibilité d'absence pour formation continue (13). 
La cancérologie dermatologique est la spécialisation la plus pratiquée avec $35 \%$ de notre population. Cela reflète le dépistage des tumeurs cutanées, la prise en charge chirurgicale d'exérèse de ces tumeurs, la prescription du bilan d'extension et le suivi régulier plutôt que les traitements des stades avancés (seuls $19 \%$ de notre série sont $\mathrm{PH}$ à temps plein). Le vieillissement de la population est corrélé à la durée d'exposition solaire et ainsi à l'augmentation de l'incidence des cancer cutanés (14).

Chaque année, 42 dermatologues passent le DU de dermatologie chirurgicale, ce qui représente sur 3 ans d'étude 4,2\% de tous les dermatologues français. Dans notre population, $25 \%$ réalisent des chirurgies complexes. La moyenne d'âge des participants à ce DU étant de 30-40 ans, il est légitime de penser que c'est par cet outil que les jeunes dermatologues développent leur activité chirurgicale.

\section{La diversification de l'activité des dermatologues se fait principalement en faveur du} laser et de la médecine esthétique, probablement par la recherche d'une rentabilité supplémentaire.

D'après une étude réalisée en 2010, seuls 3\% des internes de dermatologie en France étaient formés à la dermatologie esthétique (15). Un nouvel état des lieux réalisé en 2018 par une interne amiénoise (non encore publié) montrait que 100\% des internes recevaient la formation. Certains dermatologues se plaignant d'une trop faible valorisation de l'activité de consultation, surtout en secteur 1, se tournent vers une activité esthétique. Cette activité tend par ailleurs à continuer de se répandre parmi les dermatologues puisqu'en 2011, le CEDEF a rendu obligatoire la formation par le séminaire ADEC 1 et la réalisation de 8 demi-journées de stage en dermatologie esthétique et correctrice pendant l'internat.

En ce qui concerne les lasers, il s'agit de la dépilation et du vasculaire qui sont les plus utilisés. Ils correspondent à la demande la plus importante des patients et sont donc largement répandus et faciles à utiliser.

La moitié de notre population étudiée exerçaient déjà ou prévoyaient de pratiquer prochainement de la télémédecine. Une prise en charge tarifaire a été proposée par la CNAM (Caisse Nationale d'Assurance Maladie) et intégrée à la CCAM (Classification commune des actes médicaux) depuis le 15 septembre 2018. Seulement 6 mois après la mise en place de son remboursement par la Sécurité sociale, 7939 actes de téléconsultation ont été pris en charge. Le nombre hebdomadaire moyen de téléconsultations est passé de 200 fin 
2018 à 700 mi-février 2019 (16). Il s'agit d'une solution mise en place pour tenter de pallier à la diminution du nombre de dermatologues en France qui rend les délais de rendez-vous de plus en plus longs.

Concernant les outils de formation utilisés pendant l'internat par rapport à ceux utilisés aujourd'hui, la majorité des dermatologues diplômés se forment seuls par de la lecture scientifique et des recherches bibliographiques aux dépens des JDP, FMC/DPC et DU qui sont privilégiés par les internes étant obligés d'y assister pour se former.

Notre étude ne portait que sur une population de 3 promotions de dermatologues. Mais nous avons volontairement ciblé les jeunes dermatologues ayant fini leurs études depuis 5 à 7 ans pour avoir un groupe homogène reflétant réellement les dernières actualisations de la dermatologie. Par ailleurs, les données plus anciennes n'ont pas pu être récupérées car inexistantes et avant 5 ans d'activité, nous aurions eu une population majoritairement remplaçante ou en cours de post-internat donc difficilement interprétable.

Le biais principal de notre étude est que la population étudiée de 83 dermatologues correspond à $44 \%$ de la population concernée de 188 dermatologues. De plus, le profil des non-répondeurs est inconnu étant donné que la participation était anonyme. Il est donc difficile d'extrapoler nos résultats à toute la population cible.

De même, l'enquête menée par la FFFCEDV a obtenu 899 réponses sur 3000 dermatologues, soit seulement $30 \%$. Ce qui constitue un biais lors des comparaisons avec la population de tous les dermatologues français, les résultats de l'enquête étant eux aussi difficilement extrapolables.

D'après l'étude démographique de la dermatologie française réalisée par la FFFCDEV en 2018, 99.2\% des dermatologues français apprécient leur spécialité et $\mathbf{9 5 \%}$ la referait si c'était à refaire ( $4 \%$ choisiraient une autre spécialité et $1 \%$ ne ferait même pas médecine).

La dermatologie est la spécialité avec la plus faible prévalence de burn out (29.6\%) parmi les internes aux Etats-Unis d'Amérique avec le meilleur risque relatif : $0.60(0.39-0.88)$ et le plus bas risque absolu : -16.9 (-26.6 à -5). En ce qui concerne le regret d'avoir choisi cette spécialité, elle est la $2^{\text {ème }}$ prévalence avec seulement $1.4 \%$ d'internes américains en 
dermatologie regrettant ( $0 \%$ pour la neurochirurgie) avec un risque absolu de -6.3 (-6.8 à 1.2) et un risque relatif de 0.17 (0.02 à 1.16) (17).

\section{CONCLUSION}

Le portrait-robot du jeune dermatologue français est une femme de 33 ans ayant réalisée un clinicat puis 3 ans de remplacements à temps partiel avant de s'installer pour avoir une activité libérale de secteur II en intégrant un cabinet de médecins associés. Elle travaille environ 8 demi-journées/semaine dont plus de $75 \%$ sont consacrées à la dermatologie générale, une demi-journée/semaine de petite chirurgie et une de laser principalement de dépilatoire et vasculaire. Son activité esthétique est principalement tournée vers les peelings chimiques superficiels, l'acide hyaluronique et la toxine botulique. Elle a une cabine à UV et un appareil à PDT mais ses consultations spécialisées type cancérologie, psoriasis ou pédiatrie sont plutôt réalisées en milieu hospitalier. Elle pratique de la télémédecine et continue de se former par de la bibliographie, en assistant aux JDP, en participant à des FMC/DPC et en s'enrichissant de diplômes universitaires.

Que ce soit en dermatologie ou dans les autres spécialités, est remarquée une modification du mode d'exercice avec une croissance continue de l'exercice salariée, une féminisation de la profession médicale plus ou moins importante en fonction des spécialités et un creusement des inégalités territoriales avec des écarts de densités considérables. 


\section{BIBLIOGRAPHIE}

1. Atlas démographique 2018 du Conseil National de l'Ordre des Médecins.

2. Atlas démographique 2015 du Conseil National de l'Ordre des Médecins.

3. Newsletter 2019 des juniors en dermatologie. Collège des Enseignants de DErmatologie de France.

4. Livre blanc : les défis de la dermatologie en France. Société Française de Dermatologie. Page 20.

5. Journal Officiel du Sénat du 11/12/2014, page 2726. Disponibilité sur internet : <https://www.senat.fr/questions/base/2014/qSEQ141214114.html>

6. Journal Officiel du Sénat du 07/07/2015, page 1055. Disponibilité sur internet : <https://www.senat.fr/questions/base/2014/qSEQ141214114.html>

7. Crompton R, éditeur. Restructuring Gender Relations and Employment: The Decline of the Male Breadwinner. Oxford, New York: Oxford University Press; 1999. 254 p.

8. Crompton R, Sanderson K. Credentials and careers: some implications of the increase in professional qualifications amongst women. Sociology. 1986; 20:25-42.

9. L’emploi du temps des médecins libéraux. Solidarité, santé. 2010; 15:7.

10. Lapeyre N, Feuvre NL. Féminisation du corps médical et dynamiques professionnelles dans le champ de la santé. Rev Francaise Aff Soc. 2005 ;59-81.

11. Aynaud O, Picard D. Le statut de médecin par rapport aux autres professions libérales. Rev Francaise Aff Soc. 2011; 311-21.

12. Livre vert relatif au personnel de santé en Europe.

13. Mousquès J. Le regroupement des professionnels de santé de premiers recours : quelles perspectives économiques en termes de performance? Rev Francaise Aff Soc. 2011; 253-75.

14. Epidémiologie des cancers cutanés - Détection précoce des cancers de la peau. Institut National du Cancer.

15. Plee J, Barbe C, Richard M-A, Dreno B, Bernard P. [Survey of post-graduate training for Dermatology and Venereology residents in France (2005-2010)]. Ann Dermatol Venereol. $2013 ; 140: 259-65$.

16. Télémédecine : 8000 consultations remboursées par la Sécu en six mois - L’Express, 2019.

17. Dyrbye LN, Burke SE, Hardeman RR, Herrin J, Wittlin NM, Yeazel M, et al. Association of Clinical Specialty With Symptoms of Burnout and Career Choice Regret Among US Resident Physicians. JAMA. 2018; 320:1114-30. 


\section{ANNEXES}

Annexe 1 : Le livret de l'interne de 2007 


\section{Annexe 2 : Le questionnaire}

1) De quel sexe êtes-vous ?
a. Homme
b. Femme

2) Quel âge avez-vous ?

3) En quelle année avez-vous terminé votre internat ?

4) Avez-vous fait un post-internat ?
a. Chef de clinique Assistant
b. Assistant
c. Non

5) Dans quel CHU avez-vous été formé pour votre DES ?

6) Quelles ont été vos formations complémentaires pendant votre cursus DES ?
a. Diplômes (inter)universitaires
b. Master 1
c. Master 2
d. Participation à des FMC locales, régionales ou nationales
e. Réunions de la Société Française de Dermatologie
f. JDP (Journées Dermatologiques Parisiennes)
g. JNDP (Journées Nationales de Dermatologie Provinciales)
h. JIRD (journées interactives de réalités thérapeutiques)
i. Congrès du GERDA (Groupe d'Études et de Recherche en Dermato- allergologie)
j. Cours de dermatologie pédiatrique d'Arcachon
k. Séminaire de dermatologie pédiatrique de l'hôpital Necker
1. Journée du Groupe Dermatologie esthétique et correctrice de la SFD
m. Journées du Groupe Laser de la SFD
n. Journées du Groupe Dermatologie chirurgicale de la SFD
o. Séminaire d'angiodermatologie la SFD
p. Congrès de la Société Française de Photo-Dermatologie
q. Congrès de la Société de Recherche Dermatologique (CARD)
r. Congrès internationaux : EADV, AAD, ESDR, congrès mondial
s. Compagnonnage : stage chez des collègues pour apprendre une ou plusieurs technique(s)
t. Formations à distance par internet
u. Bibliographie, lecture
v. Autres (précisez)

7) Quelle(s) formation(s) vous a (ont) éventuellement manqué lors de votre cursus de DES ?

8) Exercez-vous aujourd'hui dans la région du CHU où vous avez été formé ?
a. Oui
b. Non : pourquoi ?
i. Retour à la région d'origine 

ii. Suivi du conjoint
iii. Opportunité d'un poste ailleurs
iv. Envie de changement
v. Autre (précisez)

9) Exercez-vous dans :
a. Dans une ville de $\mathrm{CHU}$ ou sa banlieue
b. Dans une autre ville de 30000 à 100000 habitants
c. Dans une ville de moins de 30000 habitants

10) Votre zone d'exercice est-elle considérée comme un désert médical : oui / non

11) Quel est votre statut? (Plusieurs réponses possibles)
a. Activité libérale
b. Collaborateur libéral
c. Vacations hospitalières
d. Vacations dans un centre de santé
e. Remplaçant en médecine libérale
f. Praticien hospitalier temps plein
g. Praticien hospitalier temps partiel
h. Statut universitaire : PHU, MCU-PH, PU-PH
i. Salarié dans un centre de santé
j. Salarié dans l'industrie pharmaceutique
k. Autre :...

12) Où travaillez-vous ? Plusieurs réponses possibles.
a. Cabinet libéral
b. Clinique
c. $\mathrm{CH}$ périphérique
d. $\mathrm{CHU}$
e. Centre de santé
f. Industrie pharmaceutique
g. Autres activités : ...

13) Avez-vous fait des remplacements ?
a. Non
b. Oui
i. Combien de temps en années?
ii. Temps plein
iii. Temps partiel
iv. En statut d'auto-entrepreneur

14) Quelle part de votre activité représente la dermatologie générale (non esthétique) ?
a. $<20 \%$
b. $20-50 \%$
c. $50-75 \%$
d. $>75 \%$

15) Combien de demi-journées travaillez-vous par semaine ? 
16) Réalisez-vous des consultations spécialisées
a. Allergologie
b. Angiologie
c. Cancérologie
d. Cuir chevelu
e. Malformations vasculaires
f. Dermatologie esthétique
g. Médecine interne et connectivites
h. Muqueuse buccale
i. Muqueuse génitale
j. Photobiologie
k. IST
1. Consultations spécialisées VIH
m. Ongle
n. Peau noire
o. Pédiatrie
p. Psoriasis
q. Troubles pigmentaires
r. Troubles trophiques des membres inferieurs
s. Autre : préciser

17) Ces consultations spécialisées sont-elles réalisées
a. A votre cabinet
b. En milieu hospitalier
c. Ailleurs (préciser)

18) Quelles techniques utilisez-vous ?
a. Chirurgie
b. Cryochirurgie
c. Laser
d. PDT
e. Photothérapie UVA et/ou UVB
f. Tests allergologiques
g. Techniques esthétiques
h. Echographie/ Doppler
i. Sclérose de varices
j. Capillaroscopie
k. Hypnose

19) Faites-vous de la chirurgie dermatologique?
a. Non
b. Oui :

i. Combien de demi-journées par semaine (ou équivalent $1 / 2$ journées) ?

ii. Petite chirurgie uniquement

iii. Exérèse nécessitant un acte de reconstruction par greffe cutanée ou lambeau

20) Faites-vous du laser?
a. Non
b. Oui : 
i. Combien de demi-journées par semaine (ou équivalent $1 \frac{1}{2}$ journées) ?

ii. Vasculaire :

1. Colorant pulsé

2. KTP

3. Yag

4. Diode, lumière pulsée

iii. $\mathrm{CO} 2$

1. Ablatif continu

2. Fractionné

3. Non ablatif

iv. Erbium

v. Dépilatoire :

1. Alexandrite

2. Nd-Yag

vi. Pigmentaire :

1. Yag

2. Q-switched

vii. Lumière intense pulsée (IPL)

viii. LED (Light Emitting Diode)

ix. Radiofréquence

21) Faites-vous d'autres techniques esthétiques (en dehors du laser esthétique) ?

a. Non

b. Oui :

i. Combien de demi-journées par semaine (ou équivalent $1 / 2$ journées) ?

ii. Cryolipolyse

iii. Filler (acide hyaluronique ou inducteur tissulaire)

iv. Fils permanents/ résorbables sous-cutanés à cônes ou à crans

v. Laser

vi. Micro-greffes de cheveux

vii. Micro-needling

viii. Peeling

1. Superficiel

2. Moyen

ix. Plasma riche en plaquettes

$\mathrm{x}$. Toxine botulique

xi. Greffe mélanocytaire

xii. Maquillage et tatouages médicaux

xiii. Phlébologie esthétique (microsclérose)

22) En raison des modifications récentes de la CNAM intégrées à la CCAM, pratiquezvous la télémédecine ou prévoyez-vous d'en pratiquer prochainement?
a. Non
b. Télé-expertise comme expert
c. Télé-expertise comme requérant
d. Télé-consultation

23) Actuellement, quelles formations privilégiez-vous ?

a. Diplômes (inter)universitaires 
b. Participation à des DPC (Développement Professionnel Continu)

c. JDP (Journées Dermatologiques Parisiennes)

d. JNDP (Journées Nationales de Dermatologie Provinciales)

e. JIRD (journées interactives de réalités thérapeutiques)

f. Congrès internationaux : EADV, AAD, ESDR

g. Journées des sous-groupes de la SFD

h. 4 saisons de la SFD

i. Vacations hospitalières

j. Compagnonnage : Stage chez des collègues pour apprendre une ou plusieurs technique(s)

k. Formations à distance par internet

1. Bibliographie, lecture

m. Aucune

n. Autres (précisez)

24) Si activité libérale :
a. Secteur 1
b. Secteur 2
c. Non conventionné

25) Si activité libérale, avez-vous :
a. Repris le cabinet d'un dermatologue parti en retraite
b. Ouvert votre propre cabinet tout seul
c. Ouvert un cabinet avec des dermatologues associés
d. Intégré un cabinet de dermatologues associés
e. Intégré une maison médicale
f. Intégré une maison de santé pluridisciplinaire
g. Autre

26) Si activité libérale, combien de temps après la fin de votre internat (en années) vous êtes-vous installé ?

27) Si vous êtes installé : avez-vous rencontré des difficultés pour votre installation ?

28) Si vous ne l'êtes pas : quels sont les freins à votre installation?
a. Pas envie
b. Craintes des charges
c. Difficultés de gestion d'une entreprise
d. Manque d'informations
e. Manque financier
f. Manque de formation à la chambre de commerce et d'industrie

29) Souhaitez -vous que vous soient communiqués les résultats de cette enquête ?

Si oui, vous acceptez la perte d'anonymat pour l'envoi des résultats :

Votre adresse mail ici.

30) Autres commentaires éventuels à nous adresser. 